Check for updates

Cite this: Mater. Chem. Front. 2020, 4, 472

Received 24th September 2019 Accepted 19th November 2019

DOI: 10.1039/c9qm00592g

rsc.li/frontiers-materials

\section{Spectroscopic studies of the optical properties of carbon dots: recent advances and future prospects}

\begin{abstract}
Qingnan Zhao, Wei Song, (D) * Bing Zhao (D) and Bai Yang (D)
Carbon dots (CDs) are a new class of luminescent carbon nanomaterials possessing small size, excellent water solubility, low toxicity, tunable light-emitting properties and good biocompatibility, which display bright prospects in catalysis, drug delivery, bioimaging, biosensing and energy conservation and storage. In the past several years, great achievements on the synthetic strategies, characterization techniques, unique optical properties and novel applications of a large variety of CDs have been realized. In this review, the brilliant optical properties of CDs-based nanomaterials via a series of spectroscopic techniques are summarized. After a brief introduction of the characteristic of CDs, we will focus on the optical properties of the CDs, including photoluminescence (PL), near-infrared (NIR) fluorescence, surface-enhanced Raman scattering (SERS), phosphorescence, chemiluminescence (CL), electrochemical luminescence $(E C L)$, and chirality. The correlation between the structure of CDs and their optical properties has been discussed. In addition, some challenges and future prospects in the field of the optical properties of CDs have been highlighted. With the rapid development of their superior optical properties, it is anticipated that CDs will be applicable in a large number of research fields.
\end{abstract}

\section{Introduction}

Carbon, one of the richest elements in the universe, usually possesses a number of interesting allotropes. Carbon family materials are mainly composed of fullerenes, ${ }^{1,2}$ carbon nanotubes, ${ }^{3-5}$ diamonds, ${ }^{6-8}$ graphene, ${ }^{9,10}$ graphdiyne ${ }^{11,12}$ and carbon dots

State Key Laboratory of Supramolecular Structure and Materials, Jilin University, Changchun, 130012, P. R. China. E-mail: weisong@jlu.edu.cn
(CDs). ${ }^{13-15}$ Among these carbon materials, CDs show the smallest size and superior hydrophilic properties, and exhibit promising applications in catalysis, drug delivery, bio-imaging, bio-sensing and energy devices. CDs were firstly discovered in 2004 by Scrivens and co-workers from a crude suspension during the separation and purification of single-walled carbon nanotubes using a gel electrophoresis method. ${ }^{16}$ In 2006, Sun and co-workers proposed a chemical synthetic strategy to fabricate luminescent carbon nanoparticles which are called "carbon

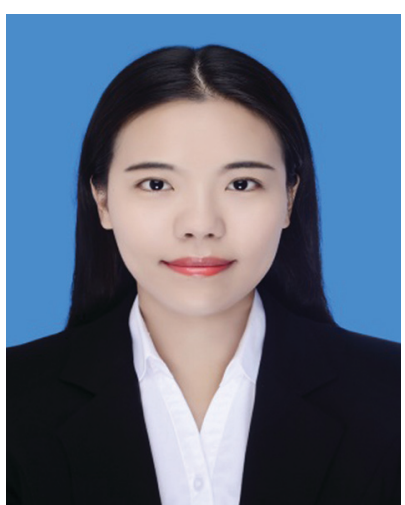

Qingnan Zhao
Qingnan Zhao is now a PhD student at the State Key laboratory of Supramolecular Structure and Materials in Jilin University, majoring in physical chemistry. She obtained her BS degree from Northeast Petroleum University in 2016 and MS degree from the Department of Analytical Chemistry at Jilin University in 2019. Her research interests are related to the surface-enhanced Raman scattering technique for catalysis and detection based on multifunctional composite nanomaterials.

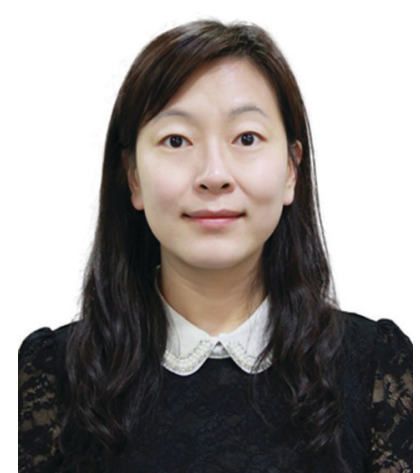

Wei Song
Wei Song is a Professor of the State Key laboratory of Supramolecular Structure and Materials at Jilin University. She received her $B S$ degree from Beihua University in 2003 and $P h D$ degree in physical Chemistry from Jilin University in 2008. Her current research focuses on the fabrication of new functional surface-enhanced Raman scattering substrates with catalytic properties for biological and sensing applications. 
quantum dots" (CQDs). ${ }^{17}$ Afterwards, a large number of researchers have devoted their efforts in this field and developed many new synthetic approaches to prepare CDs with varied compositions and chemical structures as well as optical properties. These CDs have shown great promising applications in light-emitting diodes (LEDs), catalysis, sensing, biotechnology, energy devices, etc. ${ }^{18-23}$ Recently, to avoid energy consumption and environmental pollution, an energy-efficient and sustainable strategy has been developed to prepare green carbon nanodots (CNDs) and yellow graphene quantum dots (GQDs) as well as green CQDs by using different reactants as carbon sources at room temperature, which showed a broad application prospect for bio-imaging, bio-labelling and bio-analysis. ${ }^{24,25}$

Generally, most of the CDs show a size of less than $10 \mathrm{~nm}$ and intrinsic fluorescence properties. The chemical structure of CDs is made up of nitrogen/polymeric aggregations/oxygenbased groups (e.g., $\mathrm{C}=\mathrm{O},-\mathrm{OH},-\mathrm{COOH}, \mathrm{C}-\mathrm{N}, \mathrm{C}-\mathrm{O}$ ) and $\mathrm{sp}^{2} / \mathrm{sp}^{3}$ hybrid conjugated carbon. ${ }^{26-29}$ Typically, CDs are primarily classified into GQDs, CNDs and CQDs, which present different chemical structures and optical properties, respectively. ${ }^{18,30-32}$ GQDs are a few layers of graphene sheets with lateral dimensions of less than $10 \mathrm{~nm}$. The zero-dimensional (0D) GQDs converted from two-dimensional (2D) graphene sheets display anisotropism, quantum-size effect and exciton confinement. The conjugated $\pi$-domains and the edge structure of the GQDs contribute to their unique luminescence properties. In addition, GQDs usually show favorable crystallinity. Different from GQDs, CNDs are a type of ultrasmall carbon nanoparticles with a spherical morphology and usually an amorphous lattice structure. CNDs are composed of cross-linked or aggregated monomers and linear polymers. However, some CNDs are not entirely linear polymers or disordered monomers owing to the existence of hexagonal carbon networks in amorphous CNDs. CQDs are composed of multiple layers of graphitic sheets and possess a spherical crystalline structure. In addition to typical GQDs, CNDs and CQDs, carbonized polymer dots (CPDs) have also been put forward to verify the vital role of the polymerization and carbonization processes in the synthesis of CDs in recent years. ${ }^{33}$ So far, a large variety of approaches have been developed to synthesize CDs with different compositions and chemical structures, which are mainly divided into top-down methods based on cutting different carbon materials (e.g., carbon fiber, graphene oxide (GO), fullerenes and graphite electrodes) and bottom-up methods that are generally involved in the organic synthesis of small molecules, self-assembly of polycyclic aromatic hydrocarbons and carbonization of carbohydrates. ${ }^{34-37}$

Many characterization techniques have been employed to evaluate the chemical structure of CDs, such as ultra-violet visible (UV-vis) spectroscopy, ${ }^{38,39,52}$ Fourier transform infra-red (FTIR) spectroscopy, ${ }^{40,53}$ X-ray diffraction (XRD), ${ }^{41,47}$ X-ray photoelectron spectroscopy (XPS), ${ }^{42,53}$ nuclear magnetic resonance $(\mathrm{NMR})^{43,54}$ and Raman spectroscopy ${ }^{44,51}$ (Fig. 1a-i). Typically, UV-vis absorption measurements reveal their main optical absorption in the range of $280-360 \mathrm{~nm}$ (Fig. 1a); ${ }^{52}$ however, the characteristic absorption peaks strongly depend on the preparation methods and the functional groups on the surface of CDs. ${ }^{45,46}$ XRD technology mainly provides crystallinity structure information of CDs powder, ${ }^{47}$ which contributes to high-resolution TEM analysis (Fig. 1b). FTIR spectroscopy is also employed to characterize the functional groups on the surface of CDs. ${ }^{48}$ The typical functional groups of CDs are shown in Fig. 1c, which have been investigated by FTIR spectroscopy. ${ }^{53}$ XPS is a valuable tool to confirm the elemental composition, electronic state and chemical state of the constituent elements of CDs (Fig. 1d-g). ${ }^{49,53}$ NMR can be employed to obtain the information about the surface functional groups of CDs (Fig. 1h). ${ }^{50}$ Raman spectroscopy is able to reveal the ratio of $\mathrm{sp}^{2}$ - and $\mathrm{sp}^{3}$-hybrid carbon atoms in the carbon core, which can mainly quantify and characterize the chemical structure of CDs (Fig. 1i). ${ }^{51}$ In particular, the spectral analysis is critical to evaluate the optical properties of CDs, which is beneficial to

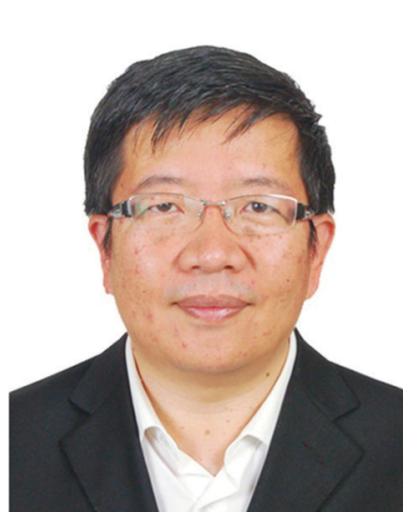

Bing Zhao
Bing Zhao received his $B S$ degree in 1984 from the Department of Chemistry, Jilin University. Afterward, he went ahead for his graduate studies at the Institute of Theoretical Chemistry, Jilin University, and obtained his MS and PhD degrees in 1987 and 1992, respectively. In 1992, he joined the chemistry faculty of the State Key Laboratory of Supramolecular Structure and Materials at Jilin University. In 1998, he was promoted to a full professor in the State Key Laboratory of Supramolecular Structure and Materials. His current research is concerned with vibrational spectroscopy and spectroscopic analysis, especially in the field of SERS spectroscopy.

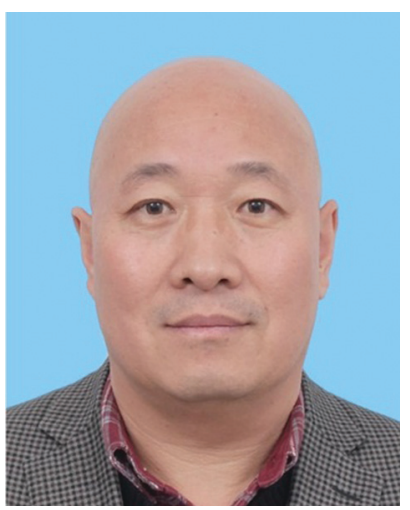

Bai Yang
Bai Yang is a professor of the State Key Laboratory of Supramolecular Structure and Materials, College of Chemistry at Jilin University. $\mathrm{He}$ received his $\mathrm{PhD}$ in polymer chemistry and physics in 1991 at Jilin University. His research interests are related to polymeric micro- and nanostructures and polymer nanohybrid functional materials, including optical, photonic, photo-electric and photo-responsive materials, and currently focus on carbon dots especially carbonized polymer dots. 

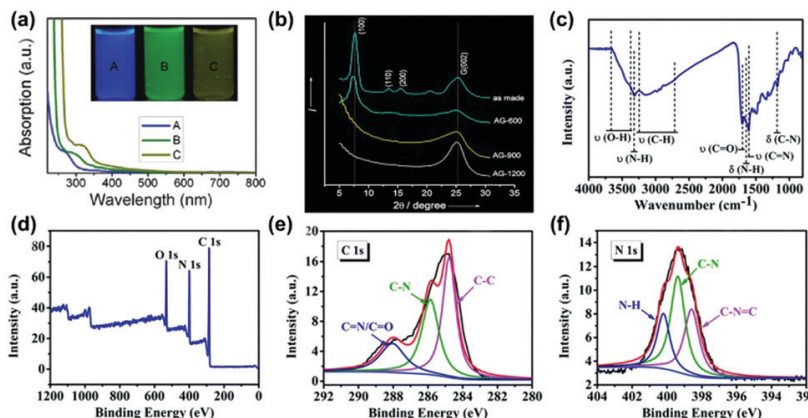

$(n$,
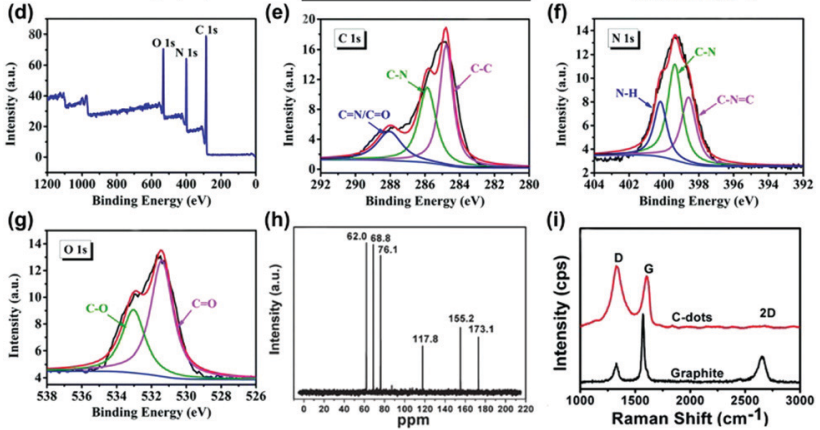

Fig. 1 Characterization techniques for the CDs: (a) UV-vis spectra of the CDs synthesized at different reaction temperatures ((A) $120{ }^{\circ} \mathrm{C}$, (B) $100{ }^{\circ} \mathrm{C}$ and $\left.(\mathrm{C}) 80^{\circ} \mathrm{C}\right) . .^{52}$ (Reprinted with permission from the American Chemical Society, Copyright 2012.) (b) Wide-angle X-ray diffraction pattern of GQDs synthesized by cyclodehydrogenation of hexaphenylbenzene (as made) and pyrolyzed at different temperatures (600, 900 and $1200{ }^{\circ} \mathrm{C}$ ). ${ }^{47}$ (Reprinted with permission from the American Chemical Society, Copyright 2011.) (c) FT-IR spectra of the CDs synthesized from folic acid and ethanol through a hydrothermal reaction and (d) XPS wide scan spectrum of the CDs, (e) C1s, (f) N1s, (g) O1s spectra. ${ }^{53}$ (Reprinted with permission from the Royal Society of Chemistry, Copyright 2019.) (h) ${ }^{13} \mathrm{C}$ NMR spectra of CDs synthesized through "polymerization" and "carbonization" from ascorbic acid. ${ }^{54}$ (Reprinted with permission from the Royal Society of Chemistry, Copyright 2012.) (i) Raman spectra ( $\lambda_{\mathrm{ex}}=633 \mathrm{~nm}$ ) of the high quality CNDs synthesized through a one-step electrochemical approach from graphite rods. ${ }^{51}$ (Reprinted with permission from the Royal Society of Chemistry, Copyright 2012.)

understand their luminescence mechanism and optoelectronic applications.

In the review, we intend to cover the optical characteristics of CDs-based nanomaterials, focusing on the development of the spectroscopic studies of CDs in photoluminescence (PL), near-infrared (NIR) fluorescence, surface-enhanced Raman scattering (SERS), phosphorescence, chemiluminescence (CL), electrochemical luminescence (ECL), and chirality. We will discuss in detail the relationship between the chemical structure and the optical properties of the CDs. Finally, we will give a brief conclusion and future prospects of CDs-based nanomaterials in applications making use of their optical properties. We hope that this review will provide valuable insights to stimulate more people to study the optical mechanism of CDs, and expand further applications of CDs in the fields of biology, catalysis, energy, and environment. Fig. 2 shows the schematic diagram of the main content of the review.

\section{Optical properties of CDs}

\subsection{PL}

PL is one of the most fascinating characteristics of CDs. CDs prepared with different approaches and materials show varied compositions and chemical structures, which can emit different

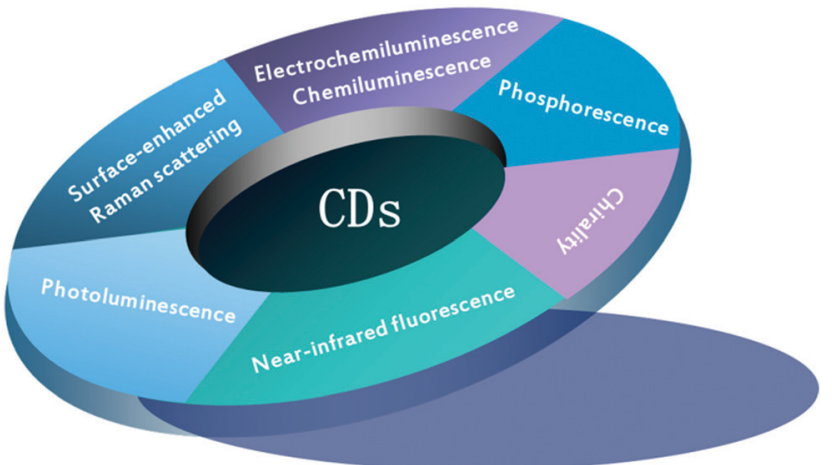

Fig. 2 Schematic diagram of the main content of this review.

colored PL, including deep ultraviolet, ${ }^{55,56}$ blue, ${ }^{57-59}$ green, ${ }^{60-62}$ yellow, ${ }^{63-65}$ red $^{66-68}$ and white light emission. ${ }^{69-71}$ Owing to their unique PL properties, the prepared CDs exhibit outstanding properties in fluorescence calibration, biomedical sensing, and light emitting devices.

2.1.1 PL mechanism. Typically, the PL of CDs may stem from the quantum size effect, surface states, and molecular states. $^{72-75}$ However, the exact PL mechanism of CDs still remains controversial and requires further investigations. The quantum confinement effect, also defined as the size effect, is one of the most acceptable mechanism models for studying the PL of CDs. In general, the energy gap of CDs is affected by the particle size and shape. Recently, some studies have focused on quantum size effects in CDs. For example, Lee and co-workers demonstrated that the strong emission of CDs was related to the quantum size of graphite fragments. Fig. 3a illustrates different emission colors and emission wavelengths
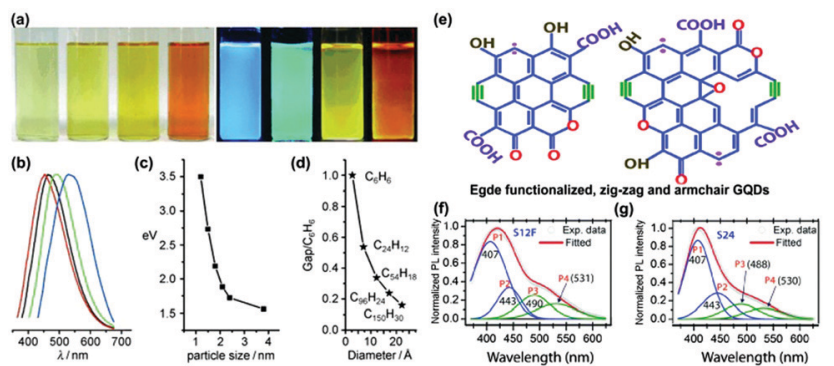

Fig. 3 (a) The optical images taken under white (left) and UV light (right) with differently sized CQDs synthesized through a one-step electrochemical approach from graphite rods; (b) PL spectra of differently sized CQDs: the red, black, green, and blue curves correspond to the PL spectra for blue-, green-, yellow-, and red-emission CQDs, respectively; (c) the relationship between the different CQDs sizes and the PL intensities; (d) relationship between the HOMO-LUMO gap and the size of the graphitic fragments. ${ }^{76}$ (Reprinted with permission from Wiley-VCH Verlag GmbH. Copyright 2010.) (e) The zigzag (magenta dot) and armchair (olive colour bonds) structures of the GQDs. (f) The PL spectra and the Gaussian peak fitting on S12F (12 $\mathrm{h}$ reaction grown functionalized GQDs sample). (g) The spectrum of the PL spectra and Gaussian peak fitting on S24 (24 h reaction grown GQDs sample). The peaks P1, P2, P3 and P4 refer to the zigzag and armchair edge states, the $\mathrm{COOH} / \mathrm{C}-\mathrm{OH}$ and $\mathrm{CQO} / \mathrm{C}-\mathrm{O}$ edge functional groups, respectively. ${ }^{81}$ (Reprinted with permission from the Royal Society of Chemistry, Copyright 2016.) 
of different sizes of CDs with a narrow size distribution. ${ }^{76}$ The relationship between PL and the size of CDs was firstly investigated through theoretical calculations. Then the authors purified the as-synthesized CDs by simple column chromatography to obtain different sizes of particles with a narrow size distribution, showing different emission colors (Fig. 3b). The relationship of the highest occupied molecular orbital (HOMO)lowest unoccupied molecular orbital (LUMO) energy gap and the size of the graphene fragments is shown in Fig. 3c. The gap gradually decreased with the increase in fragment size, which agrees with a previous study. ${ }^{77}$ The gap energy in the visible light range of graphene fragments with diameters of 1.4-2.2 nm was in agreement with the visible spectral emission of CDs with diameters smaller than $3 \mathrm{~nm}$ (Fig. 3d), illustrating that the strong emission of CDs was affected by the quantum-sized graphite structure rather than the carbon-oxygen surface. Huang and co-workers reported that the quantum size effect played an important role in the PL mechanism, and the defect state has a secondary role. ${ }^{78}$ Budyka calculated the absorption spectra of polycyclic aromatic hydrocarbons with the general formula $\mathrm{C}_{6 n}{ }^{2} \mathrm{H}_{6 n}$ serving as molecular models of GQDs. ${ }^{79}$ The transition energy decreased with increasing size $n$ according to equation $E_{1}=4.75 \times n^{-0.633} \mathrm{eV}$. The author concluded that the pure graphene sheets of fully $\pi$-conjugated $\mathrm{sp}^{2}$ systems without defects possessed sizedependent optical properties because of the quantum confinement effect. Teng and co-workers presented that the quantum confinement resulting from a size change in the $\mathrm{sp}^{2}$ domains caused a change of the $\pi^{*}-\pi$ gap rather than n-state levels. ${ }^{80}$

In addition to the quantum effect, the surface state is another important factor to determine the PL mechanism of CDs. In fact, surface states have not been accurately defined due to the diversity and uncertainty of molecular structures. The surface of CDs has abundant trap states owing to surface oxygen-related groups $(-\mathrm{COOH},-\mathrm{OH}$ and $\mathrm{C}-\mathrm{O}-\mathrm{C}$, etc. $)$, dangling bonds and hybrid carbon atoms of $\mathrm{sp}^{2}$ or $\mathrm{sp}^{3}$. Therefore, surface defect states can occur at any sites except the perfect $\mathrm{sp}^{2}$ domain. For instance, Giri and co-workers synthesized highly fluorescent and edge-controlled GQDs from different solvents. ${ }^{81,82}$ The edge of CDs mainly contains zigzag and armchair structures (Fig. 3e), providing a specific chemical structure to affect the PL property. By monitoring the PL spectra, the edge state conversion in the GQDs before and after annealing in $\mathrm{a}_{2}$ and $\mathrm{O}_{2}$ environment has been evaluated. It was found that the PL spectra of GQDs (S12F and S24) can be fitted with four Gaussian peaks (P1-P4). Typically, the blue P1 and $\mathrm{P} 2$ peaks are related to the zigzag and armchair edge defects, while the green $\mathrm{P} 3$ and $\mathrm{P} 4$ peaks are assigned to their edge oxygenated functional groups (Fig. $3 \mathrm{f}$ and $\mathrm{g}$ ).

Liu and co-workers conjectured that an energy transfer process might exist between the carbon core and the surface state. The CDs showed five emission bands at 305, 355, 410, 445 , and $500 \mathrm{~nm}$ corresponding to the five intrinsic $\mathrm{C}(4.1 \mathrm{eV})$, graphitic $\mathrm{N}(3.5 \mathrm{eV})$, pyridinic $\mathrm{N}(3.0 \mathrm{eV})$, amino $\mathrm{N}(2.8 \mathrm{eV})$ and $\mathrm{C}=\mathrm{O}(2.5 \mathrm{eV})$ related levels, respectively. ${ }^{83}$ Xiao and co-workers reported that the lifetime discrepancy of PL may not involve the $\mathrm{sp}^{2}$-related states (Fig. $4 \mathrm{a}$ and b), but the high temperature

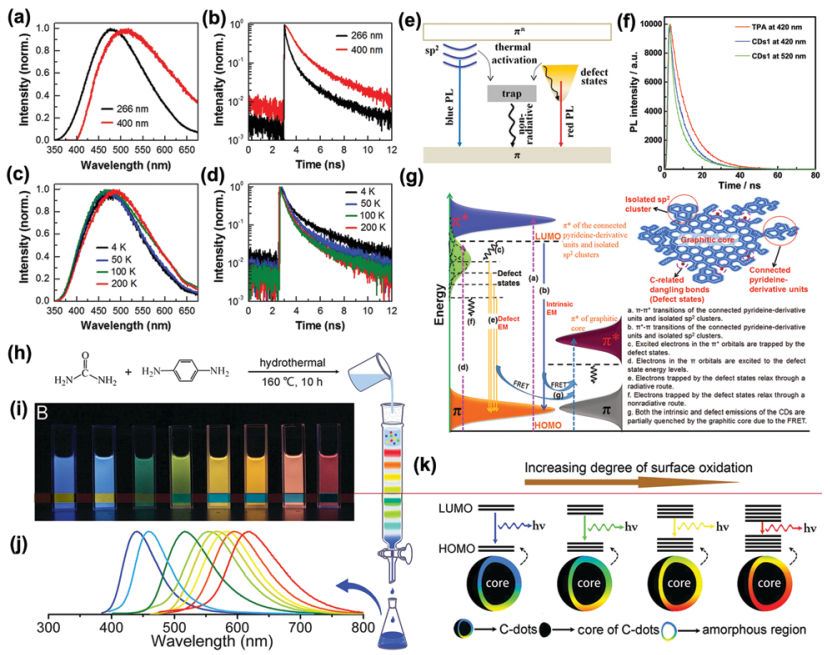

Fig. 4 (a) PL spectra of GQDs obtained by thermal deoxidization of GO sheets and (b) PL decay curves of ensemble GQDs excited at 266 and $400 \mathrm{~nm}$ by pulsed laser. (c) PL spectra and (d) PL decay curves of ensemble GQDs measured at four temperatures with pulsed laser excitation at $266 \mathrm{~nm}$. (e) Energy level structures on GQDs; energy level structures to explain the optical behaviors of photoexcited electrons in both GO sheets and GQDs. ${ }^{84}$ (Reprinted with permission from the American Chemical Society, Copyright 2013.) (f) PL decays of free TPA (3,5-dihydro-5-oxo$2 \mathrm{H}$-thiazolo[3,2-a]pyridine-3,7-dicarboxylic acid) and CDs synthesized by thermal treating a mixture of citric acid and amino group-containing molecules at $345 \mathrm{~nm}$ laser excitation. (g) Representation of the PL mechanism of CDs. ${ }^{85}$ (Reprinted with permission from Elsevier Ltd, Copyright 2017.) (h) One-pot synthesis and purification route for CDs with distinct fluorescence characteristics. (i) Eight CDs samples under $365 \mathrm{~nm}$ UV light. (j) Corresponding PL emission spectra of the eight samples, with maxima at 440, 458, 517, 553, 566, 580, 594, and $625 \mathrm{~nm}$. (k) Model for the tunable PL of CDs with different degrees of oxidation. ${ }^{86}$ (Reprinted with permission from the American Chemical Society, Copyright 2016.).

made decay dynamics fall sharply due to nonradiative traps of defect state electrons/the $\mathrm{sp}^{2}$ (Fig. $4 \mathrm{c}$ and d). The photoexcited electrons via the $\pi-\pi^{*}$ transitions included the $\mathrm{sp}^{2}$ energy levels, the defect states and long-wavelength PL (Fig. 4e). ${ }^{84} \mathrm{Yu}$ and co-workers proposed that the PL property of CDs was the result of interactions between isolated pyridine derivative molecules and surface defect states (Fig. 4f). The PL mechanism from the defect emission of CDs was composed of some isolated $\mathrm{sp}^{2}$ clusters, connected pyridine-derivative units, abundant defect states and graphitic cores (Fig. 4g). ${ }^{85}$ Xiong and co-workers reported eight types of CDs of obvious fluorescence characteristics separated by silica column chromatography with urea and $p$-phenylenediamine as reaction materials (Fig. $4 \mathrm{~h}-\mathrm{j}$ ) and speculated that the PL from CDs surfaces were consisted of bonded oxygen atoms and conjugated carbon atoms. ${ }^{86}$ The energy gap between the HOMO and LUMO was inversely proportional to the number of incorporated oxygen species. The increment of oxidation degree resulted in a PL redshift (Fig. 4k). Some electrons and excited electrons in the p-orbital might be trapped by the defect state of energy below the $\mathrm{p}^{*}$-orbital, and then return to the holes in the p-orbital. The electrons captured by the surface defect state were eventually relaxed through radiation and non-radiation processes. The PL signal from radiation relaxation was called a defect emission. ${ }^{87}$ 
2.1.2 PL emission spectroscopic studies of CDs. One of the most significant properties of CDs is excitation-dependentemission in terms of wavelength and intensity. The PL emission spectra of CDs are roughly wide and symmetrical on the wavelength scale. In addition, the emission spectrum of CDs is affected by excitation wavelength, $\mathrm{pH}$, metal ions, solvent, solid state polymers, size and heteroatom doping (Fig. 5a-h). ${ }^{88-90}$ Aslam and co-workers studied the excitation-dependentemission PL of CDs, of which the strongest emission intensity was related to the wavelength of excitation. ${ }^{91}$ The excitationdependent-emission PL could be affected by excited electron relaxation to different energy levels as well as the surface chemistry distribution. ${ }^{91,92}$ Recently, the excitation-independentemission PL property has also been widely investigated. For example, Huang and co-workers prepared a series of CDs with different densities of amino-groups via a bottom-up approach by using citric acid (CA) as the carbon source and guanidine hydrochloride as the $\mathrm{N}$ source. It was found that the asprepared CDs with a low $\mathrm{N}$ content showed an excitationdependent-emission property; however, the samples with a high $\mathrm{N}$ content exhibited an excitation-independent-emission property. This result demonstrated that the surface states of CDs could be passivated by amino-groups, which is beneficial for achieving a high luminescence. ${ }^{93}$ Tseng and co-workers used a multiGaussian function to fit the PL spectrum of the CDs, explaining the mechanism of the excitation-dependent and excitationindependent PL, which are related to core-state emission and surface state emissive traps, respectively. ${ }^{94}$ Yong and co-workers
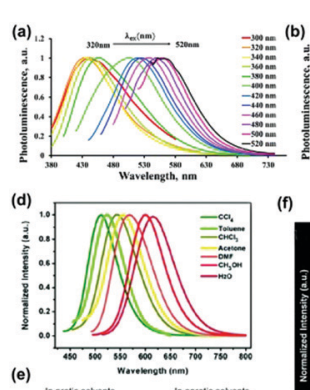

(e)

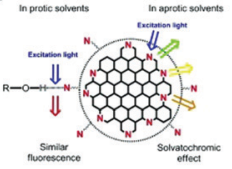

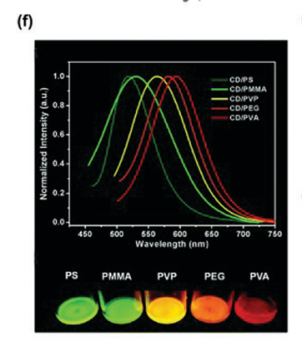
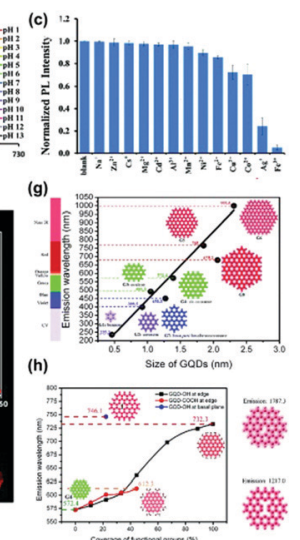

Fig. 5 (a) The excitation-dependent normalized PL for NS-CDs via a onestep facile microwave-assisted pyrolysis using citric acid and thiourea, (b) $\mathrm{pH}$-dependent $\mathrm{PL}$ spectra at different $\mathrm{pH}$ values, (c) the normalized $\mathrm{PL}$ intensity of NS-CDs in different metal ions. ${ }^{88}$ (Reprinted with permission from the Royal Society of Chemistry, Copyright 2019.) (d) The effect of solvents on the PL of CDs prepared from $p$-phenylenediamine excited by $420 \mathrm{~nm}$ light. (e) The emission processes of the CDs in different solvents. (f) PL spectra of five CDs/polymer films (excited at $420 \mathrm{~nm}$ ). ${ }^{89}$ (Reprinted with permission from the Royal Society of Chemistry, Copyright 2017.) (g) Calculated emission wavelength $(\mathrm{nm})$ of the diameter of GQDs using the time-dependent density-functional theory (TDDFT) method. The solid line is the linear fit of zigzag-edged GQDs. (h) Emission wavelength versus oxidized GQDs attached $-\mathrm{OH}$ and $-\mathrm{COOH}$ groups to edge carbon atoms. (coverage of $-\mathrm{OH}$ and $-\mathrm{COOH}$ groups from 0 to $100 \%$ ). ${ }^{90}$ (Reprinted with permission from the Royal Society of Chemistry, Copyright 2014.) demonstrated that the emission wavelength of nitrogen and sulfur co-doped carbon dots (NS-CDs) was red-shifted with increasing excitation wavelength (Fig. 5a), and the PL intensity was dependent on the $\mathrm{pH}$ value (Fig. 5b). The authors conjectured that different sizes and various functional groups on the surface of NS-CDs caused different excited state energy levels. ${ }^{88}$ Furthermore, a significant PL quenching effect was achieved in the presence of $\mathrm{Fe}^{3+}$, which could be used for $\mathrm{Fe}^{3+}$ sensing (Fig. 5c). $\mathrm{Yu}$ and co-workers reported that the solvatochromic effect was usually attributed to the intramolecular charge transfer (Fig. 5d), and proposed that the same CDs emit different colors when mixed with different polymers (solid state) under a single excitation wavelength (Fig. 5f) which come from the change of surface electronic structures. ${ }^{89}$ Using density-functional theory (DFT) and time-dependent DFT calculations, Chen and co-workers revealed that the PL of GQDs can be sensitively tuned by tailoring its size, edge configuration, shape, attached chemical functionalities, heteroatom doping and defects (Fig. $5 \mathrm{~g}$ and h). ${ }^{90}$ Doping with heteroatoms, affecting the electron levels and the overall electrical distribution, is an effective way to regulate the fluorescence properties of CDs. So far, a variety of heteroatoms (including nitrogen, boron, sulfur, tellurium, selenium, silicon, phosphorus and halogens) have been reported to be doped into CDs, which can improve their PL properties for further sensing and lightemitting applications. Jiang and co-workers analyzed the relationship between the electronegativity of heteroatom doped GQDs and their PL emission wavelength (Fig. 6a). ${ }^{95}$ It was found that the emission wavelength of the doped GQDs was associated with the electronegativity of heteroatoms. The emission wavelength of GQDs doped with $\mathrm{S}$ or Se showed a redshift because of the low electronegativity of S and Se, instead of GQDs doped with the $\mathrm{N}$ element (Fig. 6b). Xing and co-workers synthesized GQDs doped with nitrogen and chlorine with double emissions by changing the reaction time and the molar ratio of nitrogen/chlorine. ${ }^{96}$

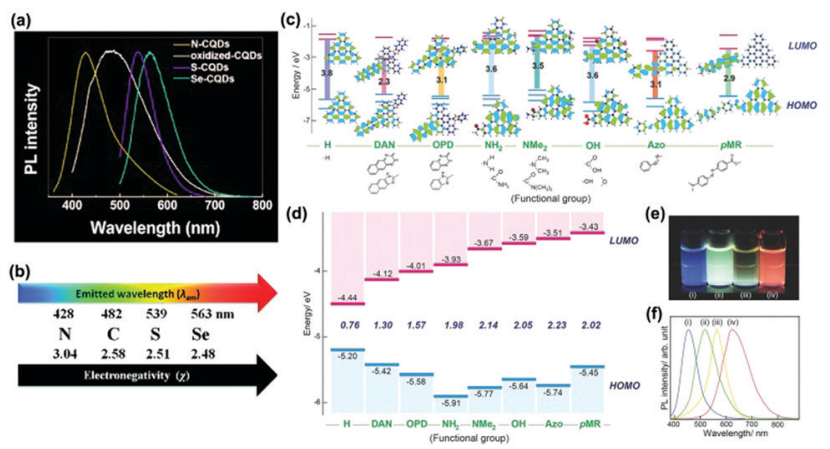

Fig. 6 (a) Normalized PL spectra of oxidized-CQDs and heteroatom (N, S, $\mathrm{Se}$ ) doped CQDs, and (b) the relationship between the electronegativity of heteroatoms and the emission wavelength of doped CQDs. ${ }^{95}$ (Reprinted with permission from the Royal Society of Chemistry, Copyright 2014.) (c) Predicted energy level diagrams for nitrogen-functionalized GQDs with different functional groups using ab initio calculations by the Gaussian 09 package. (d) Measured energy level diagram for nitrogen-functionalized GQDs. (e) PL image of nitrogen-functionalized GQDs in aqueous solution excited by a UV lamp (365 nm). (f) Normalized PL spectra (excited at $380 \mathrm{~nm}$ ) of aqueous nitrogen-functionalized GQDs. ${ }^{97}$ (Reprinted with permission from Wiley-VCH Verlag GmbH, Copyright 2016.). 
The electron transitions of nonradiative processes can happen from HOMO $\left(\mathrm{C}_{\pi}\right)$ to LUMO $\left(\mathrm{C}_{\pi^{*}}\right)$, LUMO $\left(\mathrm{N}_{\pi^{*}}\right)$ and LUMO $\left(\mathrm{Cl}_{\pi^{*}}\right)$. The excited electron at the LUMO $\left(\mathrm{C}_{\pi^{*}}\right)$ level will relax to LUMO $\left(\mathrm{N}_{\pi^{*}}\right)$ or LUMO $\left(\mathrm{Cl}_{\pi^{*}}\right)$. Thus, there were two emissions at 466 and $512 \mathrm{~nm}$, which varied with nitrogen and chlorine doping concentrations. Surface functionalization of CDs containing heteroatomic molecules is also an effective approach to obtain heteroatom-doped CDs. Tetsuka and co-workers studied the gaps and energy levels of GQDs. ${ }^{97}$ It was found that nitrogen functionalization changed the electronic structure of the system by the effective orbital resonance of doped nitrogen at the LUMO/HOMO energy levels. The authors evaluated the effects and depicted the change of the HOMO/LUMO levels of functional GQDs (Fig. 6c and d). It can be seen that GQDs with different nitrogencontaining functional groups possessed different HOMO/LUMO energy levels. Fig. 6e and f depict the PL image of nitrogenfunctionalized GQDs and their normalized PL spectra ((i) azo-GQDs, (ii) primary amine-GQDs, (iii) $o$-phenylenediamine-GQDs and (iv) diaminonaphthalene-GQDs), showing completely different color emissions and PL properties. Liu and co-workers calculated the gap energy of CDs with different types of dopant functional groups. ${ }^{83}$ As shown in Fig. 6d, the band gaps gradually increased with $\mathrm{C}=\mathrm{O}$, amino $\mathrm{N}$ and pyridine $\mathrm{N}$ groups, respectively. It could be concluded that the narrow band gaps of surface states were important for optical properties of CDs.

The quantum yield (QY) is defined as the number of emitted photons proportional to the number of absorbed photons. The QY of naked CDs is rather low (2\% to $29.9 \%$ ) because of epoxide and carboxylic groups acting as non-radiative electron-hole recombination centers, which could be determined by the fluorescence spectrum technique. Therefore, many strategies have been reported to improve the QY by changing some factors, such as the synthesis temperature, ${ }^{98}$ element doping, ${ }^{99}$ metal-enhanced fluorescence ${ }^{100}$ and surface passivation/ modification. ${ }^{101}$ Among these, element doping is one of the most efficient routes to achieve high QY. For example, Li and co-workers prepared manganese doped CDs, possessing a quantum yield of $54 \%$, which could be used to detect heavy metallic $\mathrm{Hg}^{2+}$ with a detection limit level of $\mathrm{nM} .{ }^{102}$ Yang and co-workers synthesized nitrogen/silicon co-doped CQDs (N, Si-CQDs) with a high QY of $97.3 \%$ through a hydrothermal approach, which had great potential to be applied in white LEDs. ${ }^{103}$

The PL decay lifetime reflects the time of the electrons in the excited state before going back to the ground state, which plays a vital role in practical applications in sensing, optoelectronics and bio-imaging. ${ }^{104}$ The PL decay lifetime is usually influenced by the concentration of reporter, excitation laser power and even temperature conditions. For example, Zbořil and co-workers prepared N,S-co-doped CDs (N,S-CDs) and found that the PL lifetime reduced from 11.0 to $5.3 \mathrm{~ns}$ with the temperature increasing from 2 to $80{ }^{\circ} \mathrm{C} .{ }^{105}$ This result demonstrated the potential applications of the N,S-CDs for thermal sensing.

In a word, the unique PL properties of varied types of CDs have been investigated in recent years. It is of great importance to reveal the PL mechanism from steady PL spectroscopy. In addition, the unique chemical and electronic structure of CDs contributes to the high QY and long PL decay lifetime. However, some new spectroscopic measurements are necessary to clarify the PL properties of CDs.

\subsection{NIR fluorescence}

So far, scientists have taken great efforts to synthesize various types of CDs with different strategies. The optimal excitation wavelength of most CDs is in the UV range, which limits their broad applications in biomedical fields. On the other hand, near-infrared emissive CDs (wavelength $>650 \mathrm{~nm}$ ) can decrease autofluorescence and penetrate a bio-sample. Therefore, NIR emitting CDs are very suitable for optical bio-imaging. For example, Hao, Zeng and co-workers developed novel NIR-II-emitting CDs excited with a $808 \mathrm{~nm}$ laser, which possessed a high quantum yield at 900-1200 $\mathrm{nm}$ and excellent biocompatibility. Furthermore, the prepared CDs showed high photothermal efficiency (30.6\%), and were successfully applicable in in vivo NIR-II bioimaging (Fig. 7a). ${ }^{106}$ The results showed that the tumor after intratumoral injection (i.v.) and intravenous injection (i.t.) of CDs disappeared on the sixth day compared with injection of PBS solution (Fig. 7b-e).

Recently, Qu, Rogach and co-workers reported CDs with remarkable two-photon-induced NIR emission as well as three-photon-induced red emission in dimethyl sulfoxide. ${ }^{107}$ The sulfoxide/carbonyl connected to the edges and the outer side of the CDs led to an increase of the discrete energy levels and degree of surface oxidation. Moreover, the functionalized

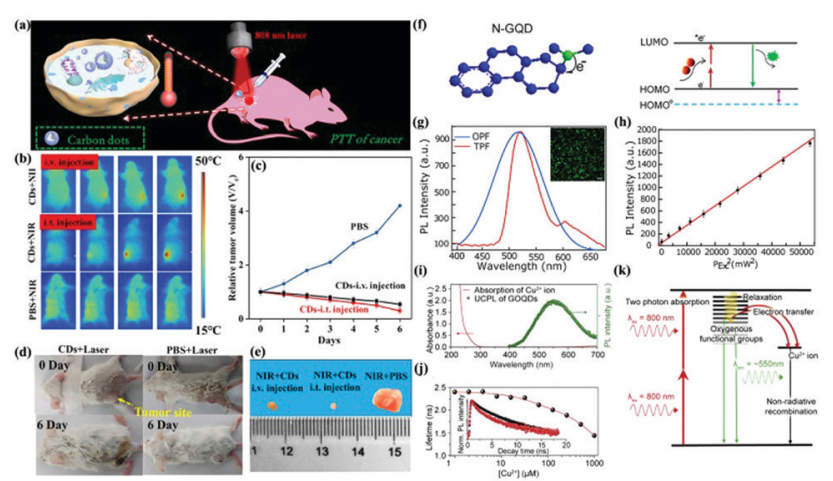

Fig. 7 (a) Schematic diagram of photothermal therapy of cancer in vivo using NIR-II emission of CDs. (b) Representative infrared thermal images of tumor-bearing mice under $808 \mathrm{~nm}$ laser irradiation. (c) Time course change in the relative volume after treatment by using CDs and PBS. (d) Digital photographs of the tumor-bearing mice after different treatments. (e) Photographs of tumors harvested from mice after 6 days of treatment. ${ }^{106}$ (Reprinted with permission from the American Chemical Society, Copyright 2019.) (f) Fluorescence mechanism between a $\pi$-conjugated system and N-GQDs. (g) Two-photon-induced fluorescence spectrum of N-GQDs solution under $800 \mathrm{~nm}$ femtosecond laser excitation. (h) Quadratic relationship of the fluorescence intensity of the N-GQDs aqueous solution with different excitation laser powers at $800 \mathrm{~nm}^{110}$ (Reprinted with permission from the American Chemical Society, Copyright 2013.) (i) UV-vis absorption spectrum of $\mathrm{Cu}^{2+}$ ions and the PL spectrum of GOQDs excited by a wavelength of $800 \mathrm{~nm}$. (j) PL lifetime of the GOQDs depending on the $\mathrm{Cu}^{2+}$ concentration. (k) GOQD quenching modeling via electron transfer from the UCPL GOQDs to the $\mathrm{Cu}^{2+}$ ions. ${ }^{113}$ (Reprinted with permission from Elsevier Ltd, Copyright 2014.) 
CDs promoted the NIR absorption band and enhanced NIR luminescence, leading to their potential applications in NIR fluorescence imaging. Recently, $\mathrm{Wu}$, Xiao and co-workers synthesized N-doped CDs through a solvothermal reaction, exhibiting NIR emission with two photon luminescence. ${ }^{108}$ In addition, the prepared $\mathrm{N}$-doped $\mathrm{CDs}$ displayed a piezochromic luminescence with reversibility, showing a red- and blue-shifted PL with applied pressure increasing from 0.07 to $1.70 \mathrm{GPa}$ and a red- and blue-shifted PL with an external pressure from 5.18 GPa to $1 \mathrm{~atm}$, which could expand their applications to detect external pressure stimuli.

Upconversion PL is an anti-Stokes type emission which exhibits emission light of shorter wavelength (single photon of higher energy) through absorption of two or more photons of longer wavelength. Then two- or multi-photon fluorescence properties can be achieved in the near-infrared region, demonstrating promising applications in the biomedical imaging field. In 2007, Sun and co-workers first reported up-converted CDs with two-photon fluorescence, which was excited using an $800 \mathrm{~nm}$ femtosecond infrared pulsed laser. ${ }^{109}$ From then on, many researchers have focused on this field. For example, $\mathrm{N}$-doped GQDs (N-GQDs) have been synthesized from GO in dimethylformamide solution via a solvothermal reaction, showing excellent two-photon fluorescence properties due to the rigid plane and large $\pi$-conjugated system of $N$-GQDs (Fig. 7f). Fig. $7 \mathrm{~g}$ (the inset is a fluorescence image of N-GQDs) shows the fluorescence spectrum of N-GQDs solution induced by an $800 \mathrm{~nm}$ wavelength femtosecond laser. ${ }^{110}$ The PL intensity had a quadratic relationship with the excited laser power (Fig. 7h). It was found that two-photon fluorescence with a much narrower bandwidth than the one-photon fluorescence spectrum was obtained. Photon absorption with NIR excitation contributes to the green fluorescence of the prepared N-GQDs. Cui and co-workers demonstrated a facile strategy to enhance the upconversion PL and tailor the red-shift fluorescence of CDs via the introduction of $\mathrm{H}_{2} \mathrm{O}_{2}$ during a hydrothermal reaction. ${ }^{111}$ It could be considered that the upconverted PL property resulted from a multiphoton active process.

The upconverted PL property enabled CDs promising applications in sensing, catalysis, and bio-imaging fields. Recently, highly fluorescent N-doped CDs have been prepared through a solar irradiation strategy between $o$-phenylenediamine and citric acid, exhibiting not only ultra-violet $(378 \mathrm{~nm})$ pumped emissions but also up-converted PL signals in the NIR range $(750 \mathrm{~nm}) .{ }^{112}$ The prepared $\mathrm{N}$-doped CDs provides an efficient multi-modal sensing platform for $\mathrm{Hg}^{2+}$. Cho, Seo and co-workers explored quenching properties of upconversion PL on graphene oxide quantum dots (GOQDs) and $\mathrm{Cu}^{2+}$ ions (Fig. 7i-k). ${ }^{113}$ It was found that the PL quenching was attributed to an electron transfer instead of an energy transfer mechanism. In recent years, up-converted CDs-based hybrids have aroused a lot of attention for their promising applications in photocatalysis and cell imaging. ${ }^{114-117}$ For instance, the photocatalytic activity of rutile $\mathrm{TiO}_{2} /$ GQDs hybrids for methylene blue degradation under visible light was significantly improved compared with individual rutile $\mathrm{TiO}_{2}$ nanoparticles and bare
GQDs. Furthermore, the photocatalytic activity of the rutile $\mathrm{TiO}_{2} /$ GQDs was also higher than that of anatase $\mathrm{TiO}_{2} /$ GQDs. The superior catalytic efficiency of the rutile $\mathrm{TiO}_{2} / \mathrm{GQDS}$ was owing to the upconverted PL peak of GQDs (407 nm) under visible light irradiation with an energy $(3.05 \mathrm{eV})$ larger than the band gap of rutile $\mathrm{TiO}_{2}(3.0 \mathrm{eV})$ but smaller than that of anatase $\mathrm{TiO}_{2}(3.2 \mathrm{eV}) .{ }^{114}$ Another example of novel CQDs modified $\mathrm{Bi}_{20} \mathrm{TiO}_{32}$ showed a rate constant 4.3 times higher than that of pure $\mathrm{Bi}_{20} \mathrm{TiO}_{32}$ for the photodegradation of isoproturon, which was attributed to the function of CQDs as an up-conversion photosensitizer. ${ }^{115}$ Recently, CDs-based hybrids have also been designed for two-photon biosensing applications. Tian and co-workers for the first time reported the preparation of a CDs-based inorganic-organic hybrid system for imaging and biosensing of $\mathrm{pH}$ in tumor tissue, e.g. mouse LLC-MK2 cells and human A549 cells at a depth of 65-185 $\mu \mathrm{m}$ through twophoton microscopy, demonstrating the practical applications of CDs in living cells. ${ }^{116}$

\subsection{SERS spectroscopy}

SERS is a non-invasive spectroscopic technique based on molecular vibrational fingerprints that quantifies and identifies ultra-trace analytes. ${ }^{118,119}$ Generally, plasmonic nanostructures composed of $\mathrm{Ag}, \mathrm{Au}, \mathrm{Cu}$ and $\mathrm{Al}$ have been employed as SERS substrates to boost Raman signals, which originates from two mechanisms: (1) electromagnetism (EM) based on surface plasmon resonance (SPR) on the metal surface, ${ }^{120}$ (2) charge transfer (CT) prompted by the interaction between the metal surface and adsorbed molecules. ${ }^{121}$ The development of SERS is associated with progress of the fabrication of novel substrates. Thus, the manufacture of efficient SERS substrates becomes more vital in this study field. The "hot spots" existing in the nanogap between metal nanoparticles is considered to be a key issue to produce outstanding SERS substrates. ${ }^{122}$ CDS without doping and modification were first regarded as SERS substrates by Shi and co-workers in 2012. ${ }^{123}$ An electrophoresis deposition strategy was developed to assemble GQDs into nanotube arrays by using a nanoporous anodic aluminum oxide membrane as a template. By using Rhodamine 6G (R6G) molecules as probes, the highest 74-fold SERS enhancement could be obtained on the surface of GQDs nanotubes, which was much higher than those on the graphene substrate. The SERS spectra revealed that the detection limit could reach around $10^{-9} \mathrm{M}$, demonstrating their high sensitivity. In addition to dye probes, this SERS substrate has also shown the capacity for 2,4-dinitrotoluene (2,4-DNT) detection. The SERS spectrum showed similar vibrational frequencies to those previously reported $\mathrm{Au}$ and $\mathrm{Ag}$ substrates. Similar to the graphene film, efficient CT between GQDs and target molecules should contribute to the excellent SERS property of the GQDS nanotubes. Furthermore, the unique porous structure of the GQDs nanotubes with a rough surface guaranteed the large specific surface area for target molecule adsorption as well as incident light capture, which is another key factor to generate unique SERS activity. This fact could be verified by the weak SERS signals on the surface of GQDs microbowls. 
Recently, CDs have been reported to show strong catalysis to generate gold and silver nanomaterials as efficient SERS substrates for quantitative analysis. ${ }^{124-126}$ In addition, CDs have also been used to support $\mathrm{Au}$ and $\mathrm{Ag}$ materials for efficient SERS substrates to enhance their SERS activity. For example, Qu and co-workers fabricated a honeycomb architecture composed of CQDs to support gold nanoparticles as a SERS substrate, exhibiting 8-11 times higher SERS activity than the traditional $\mathrm{Au}$ nanoparticle SERS substrate. It was speculated that the CQDs honeycomb structure and the uniformly distributed small $\mathrm{Au}$ particle size $(<10 \mathrm{~nm})$ contributed to the improved Raman response. ${ }^{127}$ Recently, our group has synthesized Ag@CDs nanoparticles (NPs) with a core-shell structure serving as SERS substrates. ${ }^{128}$ It was found that the Ag@CDs NPs could quench the fluorescence of CDs through the introduction of $\mathrm{Ag}$, which was beneficial for the SERS enhancement. In addition, the superior adsorption of target molecules by Ag@CDs also contributed to their SERS enhancement. By using $p$-aminothiophenol (PATP) as target molecules, the typical $\mathrm{a}_{1}$ vibration mode at $1077 \mathrm{~cm}^{-1}$ and $b_{2}$ vibration modes at 1143, 1390, 1434, and $1570 \mathrm{~cm}^{-1}$ were observed. The enhancement factor value was calculated to be about $6.7 \times 10^{5}$ for the Ag@CDs NP substrate. Furthermore, the prepared Ag@CDs NPs showed a better SERS enhancement than individual Ag NPs, demonstrating the synergistic effect between $\mathrm{Ag}$ and CDs for the SERS activity. More interestingly, the core-shell Ag@CDs NPs also exhibited excellent enzymatic catalytic activity towards the oxidation of $3,3^{\prime}, 5,5^{\prime}$-tetramethylbenzidine (TMB) in the presence of $\mathrm{H}_{2} \mathrm{O}_{2}$ (Fig. 8a), plasmon driven $p$-nitrothiophenol (PNTP) dimerization into 4,4'-dimercaptoazobenzene (DMAB) (Fig. 8b), and the catalytic reduction of PNTP to PATP by $\mathrm{NaBH}_{4}$ (Fig. 8c). Similarly, we have also prepared a Ag NPs/CDs hybrid via a simple chemical coupling reaction strategy as a SERS substrate to study the enhancement mechanism. ${ }^{129}$ Fig. 8d illustrates the relationship between the peak intensity ratio $\left(1144\left(b_{2}\right)\right.$, $\left.1075\left(\mathrm{a}_{1}\right) \mathrm{cm}^{-1}\right)$ of PATP molecules and the excitation energy on the surface of $\mathrm{Ag}$ NPs/CDs and Ag NPs. It could be concluded that the SERS signal of Ag NPs was associated with the polarizability of the molecules and the change of the potential under higher excitation energy, which was consistent with a previous report. ${ }^{130}$ The SERS signal of Ag NPs/CDs was related to the degree of CT. Meanwhile, the relationship between the relative intensity $\left(b_{2} / a_{1}\right)$ and the laser power of the two bands was investigated (Fig. 8e and f), from which it could be concluded that the SERS chemical enhancement mechanism was mainly controlled by the enhancement of the $b_{2}$ band.

In addition to $\mathrm{Au}$ and $\mathrm{Ag}$ hybrids with $\mathrm{CDs},{ }^{131,132}$ a $\mathrm{Cu} / \mathrm{CDs}$ hybrid has also been prepared to be an efficient SERS substrate. ${ }^{133}$ For example, Jiang and co-workers reported the fabrication of $\mathrm{Cu} / \mathrm{CDs}$ assemblies with varied morphologies through an organic-inorganic interface interaction. Owing to the lattice-matching, CDs selectively deposited on the $\mathrm{Cu}\{111\}$ plane to form a unique $\mathrm{Cu} / \mathrm{CDs}$ hybrid. Further, CDs in the $\mathrm{Cu} / \mathrm{CDs}$ hybrid not only prevented $\mathrm{Cu}$ oxidation but also provided a unique interface for optical conversion and CT, contributing to the enhanced SERS effect. The SERS detection
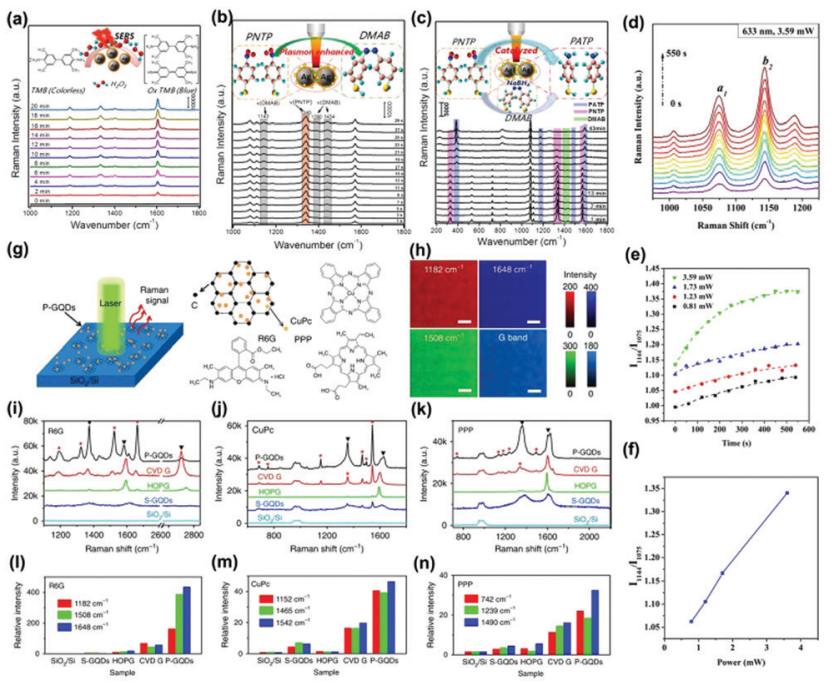

Fig. 8 Time-dependent SERS spectra of the catalytic reaction by coreshell Ag@CDs NPs. (a) The oxidation of TMB by $\mathrm{H}_{2} \mathrm{O}_{2}\left(3 \times 10^{-3} \mathrm{M}\right)$, (b) plasmondriven catalytic reaction of PNTP dimerizing into DMAB, and (c) catalyzed reduction of PNTP to PATP. ${ }^{128}$ (Reprinted with permission from the American Chemical Society, Copyright 2016.) (d) The Raman intensity of PATP molecules on Ag NPs/CDs versus time, (e) the SERS intensity ratio $\left(/_{1144} / /_{1075}\right)$ at different times with different powers, $(f)$ the SERS intensity ratio $\left(/_{1144} / /_{1075}\right)$ versus power at 300 s. $^{129}$ (Reprinted with permission from Elsevier B.V. Copyright 2017.) (g) Schematic of a P-GQDs substrate for Raman measurement and (h) Raman mapping of the intensity of the $G$ band and the characteristic peaks of R6G on P-GQDs. (i-n) Raman spectra of thermally evaporated Rhodamine 6G (R6G), copper phthalocyanine (CuPc) and Protoporphyrin IX (PPP) on $\mathrm{SiO}_{2} / \mathrm{Si}$, GQDs produced by solution processes (S-GQDs), highly oriented pyrolytic graphite (HOPG), transferred chemical vapor deposition (CVD) graphene and P-GQDs, respectively. (e-g) Relative intensity of the Raman signals of R6G, CuPc and PPP on different substrates, normalized to the signals on $\mathrm{SiO}_{2} / \mathrm{Si}^{134}$ (Reprinted with permission from Nature Publishing Group, Copyright 2018.)

limit reached $10^{-8} \mathrm{M}$ by using PATP as target molecules. The SERS enhancement mechanism of GQDs using R6G molecules as probes has been investigated by an $a b$ initio density functional theory (DFT) calculation approach. ${ }^{134}$ The CT process between GQDs and R6G would occur when their HOMO levels were close, which contributed to the SERS signal. In addition, the authors also investigated the SERS substrate based on the quasiequilibrium plasma-enhanced chemical vapor deposition GQDS (P-GQDs) grown on $\mathrm{SiO}_{2} / \mathrm{Si}$ (Fig. 8g). P-GQDs as the SERS substrate was demonstrated to be reliable via analyzing the Raman spectra (Fig. 8i-k) and relative intensity (Fig. 8l-n) of R6G, copper phthalocyanine (CuPc) and Protoporphyrin IX (PPP) on different substrates, with an ultra-low detection concentration $\left(1 \times 10^{-9} \mathrm{~mol} \mathrm{~L}^{-1}\right)$ for R6G. The authors conjectured that the superior SERS activity could be attributed to the high-quality GQDs with atomically clean surfaces and a large number of edges, as well as the enhanced CT between molecules and GQDs with appropriate diameters due to the existence of Van Hove singularities in the electronic density of states. ${ }^{134}$

\subsection{Phosphorescence}

Phosphorescence refers to a radiative transition and spinforbidden process from the triplet state $\left(T_{1}\right)$ to the singlet 
ground state $\left(\mathrm{S}_{0}\right)$. The phosphorescence lifetime is longer than the fluorescence lifetime. ${ }^{135}$ As an emerging optical response material, the room temperature phosphorescent properties of CDs have been investigated, which could be monitored by steady state PL spectroscopy and time-resolved phosphorescence spectroscopy. The first observation of phosphorescence from CDs in a polymer matrix was reported by Zhao and co-workers. ${ }^{136}$ Yang and co-workers concluded that $\mathrm{C}=\mathrm{N}$ bonds facilitate the production of triplet excitons (Fig. 9a). ${ }^{137}$ Moreover, the complex matrix was a key factor in preventing the triplet state from a long lifetime quenched by inhibiting the nonradiative processes (Fig. 9b-e). It can be seen from the optical spectra of $\mathrm{N}$ doped CDs incorporated into biuret composite matrices that they possessed a good oxygen barrier performance and the hydrogen bonds were effective in suppressing vibrational dissipation at all temperatures (Fig. 9f-i). In addition, the $\mathrm{N}$ doped CDs-based composite with distinguishable lifetime codes was expected to be applied in data encryption (Fig. 9j and k). The steady-state PL and phosphorescence spectra presented the contributions of hydrogen bonding between $\mathrm{N}$-doped CDs and biuret to the emissive triplet relaxation. The phosphorescence mechanism is ascribed to the generation of novel energy level structures from $\mathrm{C}=\mathrm{N}$ bonds on the surface of the N-doped CDs. Moreover, an ultralong phosphorescence lifetime of $1.06 \mathrm{~s}$ was achieved for the prepared N-doped CDs encapsulated in biuret under $280 \mathrm{~nm}$ excitation.
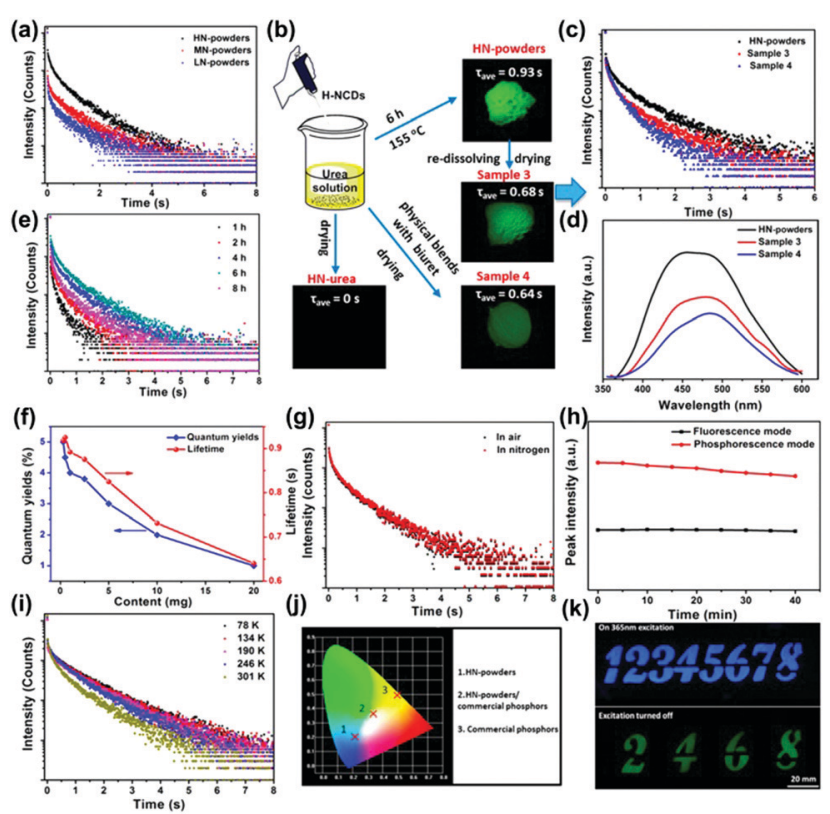

(k)

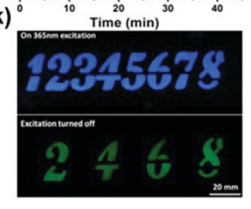

Fig. 9 (a) Lifetime decay profiles of the $\mathrm{N}$-doped CDs in composite matrices, and the emission band at $490 \mathrm{~nm}$ excited at $280 \mathrm{~nm}$. (b) Flow diagram of verification experiments. (c) Lifetime decay profiles of the emission band. (d) Phosphorescence spectra of sample powders. (e) Lifetime decay profiles of the emission band. (f) Average lifetimes and phosphorescence quantum yield (PQYs). (g) Lifetime decay profiles. (h) Repeated excitation illumination at $280 \mathrm{~nm}$ for $40 \mathrm{~min}$. (i) Lifetime decay profiles of the emission at $490 \mathrm{~nm}$. (j) Emission color coordinates of assembled LEDs. (k) Security protection applications. ${ }^{137}$ (Reprinted with permission from the American Chemical Society, Copyright 2016.)
Organic molecules have also been used to construct hydrogen-bonded networks with CDs by utilizing water molecules to promote phosphorescence emission. ${ }^{138}$ The prepared CDs-cyanuric acid (CA) hybrid displayed a high phosphorescence lifetime of $687 \mathrm{~ms}$, which was ascribed to the enhanced rigidity of the CDs after the introduction of CA. The enhancement mechanism for the phosphorescence emission of the CDs/CA system has also been investigated. ${ }^{139}$ There were two main aspects that were involved in the reducing the surface defect and increasing the degree of conjugation between the carbon core and carboxyl groups. Zhou and co-workers proposed a strategy to effectively enhance phosphorescence signals by using water molecules to build hydrogen bond networks between cyanuric acid and CDs, which effectively stiffens the $\mathrm{C}=\mathrm{O}$ bond of the CDs, as well as greatly increases the rigidity of the overall system. ${ }^{140}$

In the last few years, phosphorescence energy transfer systems between CDs and inorganic matrixes have attracted increasing attention for their promising applications for sensing and security applications. For example, phosphorescence energy transfer has been observed to take place between carbon nanotubes and Mn-doped ZnS quantum dots conjugated with capture ssDNA, which could be used for supersensitive detection of DNA. ${ }^{141}$ Similarly, the phosphorescence efficiency of CDs has been enhanced by the introduction of an inorganic layered double hydroxide $(\mathrm{LDH})$ matrix, which improved the rate of radiative attenuation from $T_{1}$ to $S_{0}$ and intersystem crossing (ISC) from $S_{1}$ to $\mathrm{T}_{n} \cdot{ }^{142}$ A molten salt (MS) strategy has been used to prepare CDs@MS, showing an excitation dependent phosphorescence property. ${ }^{143}$ The phosphorescence generation could be related to the preservation of the triplet state of CDs by the rigid structure from the crystallization of MS. The high charge density of metal ions reduced the energy gap of CDs for the achievement of ISC. It is well known that boron is an electron withdrawing atom, which is able to reduce the energy gap between the singlet and triplet states. Therefore, the introduction of boric acid into CDs was beneficial for the ISC between $S_{1}$ and $T_{1}$, which enhanced the triple excitons and the phosphorescence emission. ${ }^{144}$ The prepared heteroatom-free-CDs/boric acid hybrid displayed a yellow-green phosphorescence and an ultrahigh lifetime of $1.6 \mathrm{~s}$.

In addition to the polymers, organic molecules and functional inorganic matrices, individual CDs have also been observed to show room temperature phosphorescence. ${ }^{145-149}$ For example, Lin and co-workers proposed a self-immobilization strategy to prepare $\mathrm{N}$ and $\mathrm{P}$ doped fluorescence emissive CDs. After a further heating process, the product can convert fluorescence to ultralong phosphorescence. ${ }^{145}$ An excitation-dependent phosphorescence spectrum of the CDs powder was achieved. The steady state fluorescence and afterglow decay spectra revealed an average lifetime of about $1.39 \mathrm{~s}$. It could be concluded that the formed compact cores via a further heating step self-immobilize the excited triplet excitons due to the intraparticle hydrogen bonds. Furthermore, the $\mathrm{N}$ and $\mathrm{P}$ dopants are also beneficial to form an $\mathrm{n} \rightarrow \pi^{*}$ transition to improve the ISC process for populating triplet species. Similarly, a microwave irradiation approach has also been demonstrated to fabricate $\mathrm{N}$ and $\mathrm{P}$ doped-CDs, 
displaying the longest phosphorescence lifetime and undergoing an $\mathrm{n}-\mathrm{p}^{*}$ transition to effectively fill triplet excitons. ${ }^{146} \mathrm{~F}$ and $\mathrm{N}$ co-doped CDs (FNCDs) have also been prepared to emit green selfprotective phosphorescence with longevous triplet excited states. ${ }^{147}$ The phosphorescence of the FNCDs results from the $\mathrm{n}-\pi^{*}$ electron transitions for $\mathrm{C}=\mathrm{N} / \mathrm{C}-\mathrm{N}$ bonds as well as the reduction of the phosphorescence quenching to oxygen by the hydrogen bonds between $\mathrm{F}$ and $\mathrm{N}-\mathrm{H}$. Recently, Yang and co-workers demonstrated that a novel covalently crosslinked framework structure of the polymeric CDs could efficiently suppress the nonradiative transitions and the abundant energy levels of the polymeric CDs increased the ISC. ${ }^{148}$ Therefore, a phosphorescence emission with a broad peak centering at $494 \mathrm{~nm}$ was observed for the prepared polymeric CDs. In addition, the lifetime of the obtained polymeric CDs was calculated to be about $0.03 \mathrm{~s}$. The crosslink enhanced emission effect has been regarded as the key factor for the phosphorescence production.

\subsection{CL}

CL can be described as a process of emitting photons from the excited states to the ground states during a chemical reaction. ${ }^{150,151}$ Most properties of CL are similar to fluorescence. The difference is that the excitation energy of fluorescence comes from the excitation of external light sources, while the excitation energy of CL is produced by self-chemical reactions. In a chemical reaction, a molecule or an atom is excited by absorbing the chemical energy generated during the reaction. The energy is released by means of optical radiation when the excited molecule or atom returns to the ground state. The remarkable property of CL usually shows a high sensitivity due to the absence of an external light source. At present, the work on CL analysis based on CDs is still in its infancy.

Lin and co-workers first studied the CL properties of CDs in a mixed solution of $\mathrm{NaNO}_{2}$ and $\mathrm{H}_{2} \mathrm{O}_{2}$, which were the main emitters in the $\mathrm{CDs}-\mathrm{NaNO}_{2}-\mathrm{H}_{2} \mathrm{O}_{2}$ system. ${ }^{152}$ The CL spectra showed that the systems of $\mathrm{NaNO}_{2}+\mathrm{H}_{2} \mathrm{O}_{2}$ and CDs $+\mathrm{H}_{2} \mathrm{O}_{2}$ only exhibited a weak CL emission, while the system of $\mathrm{NaNO}_{2}+$ $\mathrm{H}_{2} \mathrm{O}_{2}+$ CDs produced a very strong CL. This result implied that the product (oxidant, $\mathrm{ONOOH}$ and $\mathrm{OH}_{3}$ ) during the reaction of nitrites and acidified $\mathrm{H}_{2} \mathrm{O}_{2}$ could transform and inject small holes of the CDs. This process, accelerating the hole-injected annihilation and increasing the number of injected electrons, brought about the release of energy in the form of $\mathrm{CL}$ emissions. ${ }^{153-156}$ Lin and co-workers also developed a CL platform based on N-doped CDs to improve the CL sensitivity in a $\mathrm{KIO}_{4}-\mathrm{H}_{2} \mathrm{O}_{2}$ system in a basic medium (Fig. 10b). ${ }^{157}$ As shown in Fig. 10a and c, the intensity (3) was much higher compared with (1) and (2) because of the decomposition of $\mathrm{H}_{2} \mathrm{O}_{2}$ and energy transfer of ${ }_{1} \mathrm{O}_{2}$ as emissive species. Dong and co-workers reported a CNDs $/ \mathrm{K}_{2} \mathrm{~S}_{2} \mathrm{O}_{8}$ system with enhanced CL efficiency induced by triethylamine (TEA) (Fig. 10f). ${ }^{158}$ The CL spectrum showed that the CL intensity of the $\mathrm{CDs} / \mathrm{K}_{2} \mathrm{~S}_{2} \mathrm{O}_{8}$ system increased after the introduction of TEA (Fig. 10d). The enhanced CL emission could result from the reduction of CNDs by the TEA free radicals to generate excited CNDs. Furthermore, the addition of CNDs at the end is beneficial to increase the CL signal (Fig. 10e). Similar to the CNDs, Abdolmohammad-Zadeh and co-workers constructed

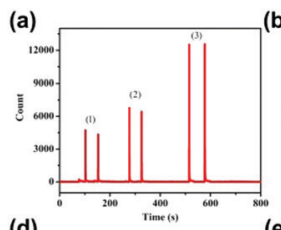

(d)

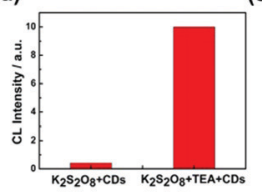

Time(s)
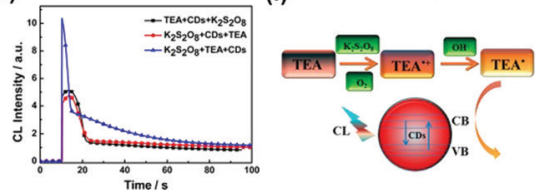

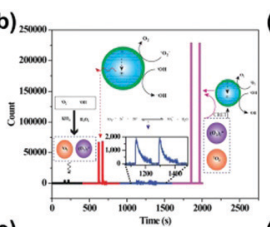

Fig. 10 (a) $\mathrm{CL}$ curves of a $\mathrm{KIO}_{4}-\mathrm{H}_{2} \mathrm{O}_{2}-\mathrm{N}$-doped CDs system ((1) $\mathrm{H}_{2} \mathrm{O}_{2}$ was added to the mixture of $\mathrm{KIO}_{4}$ and $\mathrm{N}$-CDs, (2) N-CDs was added to the mixture of $\mathrm{KIO}_{4}$ and $\mathrm{H}_{2} \mathrm{O}_{2}$, (3) $\mathrm{KIO}_{4}$ was added to the mixture of $\mathrm{H}_{2} \mathrm{O}_{2}$ and $\mathrm{N}$-CDs). (b) The mechanism of enhancement of the $\mathrm{CL}$ of the $\mathrm{KIO}_{4}-\mathrm{H}_{2} \mathrm{O}_{2}$ system by $\mathrm{N}$-doped $\mathrm{CDs}$ in acidic (the decreasing effect) and basic (increasing effect) solutions. (c) $\mathrm{CL}$ spectra of the $\mathrm{KIO}_{4}-\mathrm{H}_{2} \mathrm{O}_{2}$ system in the presence and absence of $\mathrm{N}$-doped $\mathrm{CDs}$ and $\mathrm{NaOH}\left(\mathrm{a}: \mathrm{KIO}_{4}-\mathrm{H}_{2} \mathrm{O}_{2}, \mathrm{~b}\right.$ : $\mathrm{KIO}_{4}-\mathrm{H}_{2} \mathrm{O}_{2}-\mathrm{N}$-doped $\mathrm{CDs}$, c: $\mathrm{KIO}_{4}-\mathrm{H}_{2} \mathrm{O}_{2}-\mathrm{N}$-doped $\left.\mathrm{CDs}-\mathrm{NaOH}\right)^{157}$ (Reprinted with permission from the American Chemical Society, Copyright 2016.) (d) The $\mathrm{CL}$ spectrum of the $\mathrm{CNDs} / \mathrm{K}_{2} \mathrm{~S}_{2} \mathrm{O}_{8}$ system and the CNDs/TEA/ $\mathrm{K}_{2} \mathrm{~S}_{2} \mathrm{O}_{8}$ system. (e) $\mathrm{CL}$ spectrum of the CNDs/TEA/ $/ \mathrm{K}_{2} \mathrm{~S}_{2} \mathrm{O}_{8}$ system under different reagent mixing orders. (f) The scheme of the $\mathrm{CL}$ mechanism of the CNDs/ $\mathrm{K}_{2} \mathrm{~S}_{2} \mathrm{O}_{8} /$ TEA system. ${ }^{158}$ (Reprinted with permission from the American Chemical Society, Copyright 2016.)

a graphitic carbon nitride quantum dots (g-CNQDs)- $\mathrm{K}_{3} \mathrm{Fe}(\mathrm{CN})_{6}$ CL system to achieve enhanced CL emission. ${ }^{159}$ After mixing the g-CNQDs and $\mathrm{K}_{3} \mathrm{Fe}(\mathrm{CN})_{6}$, a wide peak centered at around $503 \mathrm{~nm}$ was observed, which was similar to but significantly higher than the fluorescence band of $\mathrm{g}$-CNQDs, revealing the efficient CL property of the g-CNQDs- $\mathrm{K}_{3} \mathrm{Fe}(\mathrm{CN})_{6}$ system. Due to CL quenching in the presence of $\mathrm{Hg}$ (II) ions binding with the g-CNQDs and the CT process, this system could be used to detect $\mathrm{Hg}$ (II) with a high sensitivity and excellent selectivity. Recently, Amjadi and co-workers prepared Si doped CDs (Si-CDs) to construct a novel Si-CDs-Fe(II)- $\mathrm{K}_{2} \mathrm{~S}_{2} \mathrm{O}_{8}$ CL system, showing a CL emission peak at around $500 \mathrm{~nm} .{ }^{160}$ During the reaction, Si-CDs were used as receptors and ${ }_{1}\left(\mathrm{O}_{2}\right)_{2}{ }^{*}$ was used as a donor, producing excitedstate Si-CDs with CL emission. Besides, the group illuminated the CL system based on a CNQDs-Cu(II)- $\mathrm{H}_{2} \mathrm{O}_{2}$ system, which took place under the condition of electron and hole injection (oxygen radicals). ${ }^{161}$ This CL reaction has been utilized for the sensitive detection of $\mathrm{H}_{2} \mathrm{O}_{2}$ and glucose. Similarly, $\mathrm{N}$-(aminobutyl)- $N$-(ethylisoluminol)-GQDs- $\mathrm{H}_{2} \mathrm{O}_{2},{ }^{162} \mathrm{Cu}^{2+}-\mathrm{CDs},{ }^{163}$ and $\mathrm{Cu}(\mathrm{II}) / \mathrm{Cu}_{2} \mathrm{O} / \mathrm{N}$-doped GQDs-luminol- $\mathrm{H}_{2} \mathrm{O}_{2}$ CL systems ${ }^{164}$ have also been constructed to show superior CL properties for biosensing applications. It has been reported that the surface groups of the CDs play an important role in their CL properties. For instance, the CL intensity of CDs prepared in peroxynitrite was related to the concentration of $\mathrm{C}-\mathrm{O}$ group-related $\mathrm{O}$-states, demonstrating that ample $\mathrm{C}-\mathrm{O}$ functional groups in CDs with high O-states could promote the electron transfer. ${ }^{165}$ A recent report showed that $\mathrm{N}$-doped GQDs strongly strengthen the $\mathrm{CL}$ intensity of the permanganate-sulfite system. However, the enhancement mechanism for N-doped GQDs was not dependent on the particle size. ${ }^{166}$ The CL spectra revealed that pyridinic $\mathrm{N}$ in $\mathrm{N}-\mathrm{GQDS}$ acted as a catalytic active site to the amplified CL emission. 
With the increment of the pyridinic $\mathrm{N}$ concentration, an enhanced CL intensity was obtained.

\subsection{ECL}

ECL is a phenomenon where chemical substances that are produced on the electrode surface go through electron transfer reactions to realize excited states to generate light emission. ${ }^{167,168}$ ECL mechanisms are categorized as two types according to the source of free radicals, which are the annihilation pathway and the co-reaction pathway, respectively. ECL techniques based on the CDs materials are widely used because of their simple synthesis, low-cost equipment requirement, high sensitivity and a wide linear range. The first investigation of CDs on ECL properties was reported by Yang and co-workers in 2009. ${ }^{169}$ The CNDs were prepared through a simple microwave reaction by using poly(ethylene glycol) (PEG-200) and saccharide as the carbon sources. The ECL spectrum of the CNDs showed a similar behavior to those of traditional CdSe, CdTe, and $\mathrm{Si}$ nanocrystals. The ECL mechanism should be ascribed to the transfer of the excited CNDs $\left(\mathrm{R}^{*}\right)$ that are formed from the electron-transfer annihilation of the oxidized state $\left(\mathrm{R}^{\bullet+}\right)$ and the reduced state $\left(\mathrm{R}^{\bullet-}\right)$ under a potential cycle to the ground state via a radiative pathway, which efficiently emitted a photon. Similar to CNDs, GQDs have also been proved to be a brilliant ECL material. ${ }^{170}$ It has been reported that greenishyellow colored GQDs were prepared from a microwave reaction via cleaving GO by acids, exhibiting a maximum wavelength at around $512 \mathrm{~nm}$ in the ECL spectrum. Most importantly, the high content of $\mathrm{sp}^{2}$ carbon domains in GQDs provides their unique energy gap compared with graphene, which is beneficial for the electron transport during the ECL process. CQDs could also be assembled into nanospheres with the assistance of a surfactant (pluronic F127), showing an excellent ECL stimulus response in water, while a weak ECL signal in a nonpolar solvent. ${ }^{171}$ This is because the nonpolar CQDs (NCQDs)/F127 hybrid possesses two secondary structures, e.g., a hydrophobic layer from the polyoxypropylene (PPO) moiety of F127 and NCQDs, and a hydrophilic layer from the PPO moiety of F127. In aqueous solution, the hydrophilic layer could adsorb $\mathrm{SO}_{4}{ }^{--}$to excite nearby NCQDs, producing superior ECL emission. However, in a nonpolar solvent, the NCQDs are separated from F127, which are difficult to excite by the $\mathrm{SO}_{4}{ }^{\bullet-}$, generating weak ECL signals.

High conductive materials have been utilized to improve the ECL property as a conducting bridge for efficient electron transfer. For example, a hybrid made of sandwiched CDs labeled on secondary antibodies on a glass carbon electrode showed certain ECL signals. ${ }^{172}$ After the introduction of graphene as a conducting bridge, about 30-fold ECL enhancement was achieved. In addition to graphene, Au nanoparticles have also been used as signal transduction units for ECL amplification. Qiu and co-workers proposed a dual-potential ECL ratiometric approach to produce ECL signals by using GQDs and luminol as cathodic and anodic ECL emitters, and Au NPs as signal transduction units. ${ }^{173}$ The introduction of $\mathrm{Au}$ nanoparticles could both reduce the cathodic ECL of GQDs and increase the anodic ECL of luminol, demonstrating a dual-potential ECL ratiometric strategy to monitor kinase activity.

The ECL technique based on CDs could be used for sensing applications with a high sensitivity. For example, Wang and coworkers reported a dual-peak ECL system to detect iron ions, showing a higher efficiency than single peak ECL. ${ }^{174}$ In this system, the ECL system at $-2.84 \mathrm{~V}$ (ECL-1) was induced through an electron transfer reaction between the reduced nanoparticles and co-reactant, while the ECL system at $-1.71 \mathrm{~V}$ (ECL-2) resulted from the quenching process. In addition to iron ions, a sensitive ECL biosensor based on $\mathrm{N}$-doped CDs-Pd@Au hexoctahedra has been constructed for the detection of intracellular $\mathrm{Pb}^{2+}$ with a detection limit of $0.33 \mathrm{ng} \mathrm{mL} \mathrm{mL}^{-1}$ and relative standard deviations (RSDs) of no more than $5 \% .{ }^{175}$ Recently, a solid-state ECL sensor (CDs-Fe $(\mathrm{CN})_{6}{ }^{3-/ 4-}$ ) with an excellent signal amplification has also been constructed, which could be used to detect glutathione with a low detection limit of $54.3 \mathrm{nM}$ and excellent selectivity. ${ }^{176}$ Another example of a silicadoped GQDs-based ECL system with fluorescence was reported to detect ochratoxin. ${ }^{177}$ With the addition of concanavalin A (Con A), glucose oxidase (GO) $)_{x}-\mathrm{CeO}_{2} @ A g-G Q D s$ were also demonstrated to be used as an excellent material by further analyzing the construction of biosensors (Fig. 11a) and the ECL response of different biosensors (Fig. 11b-d) under the same conditions. This ECL system could be used to detect Con A with a high sensitivity. ${ }^{178}$ Xia and co-workers amplified dual potential ECL signals of GQDs via coupled electrochemical and chemical reactions in the presence of two different reactants $\left(\mathrm{K}_{2} \mathrm{~S}_{2} \mathrm{O}_{8}\right.$ and $\mathrm{Na}_{2} \mathrm{SO}_{3}$ ); then, CQDs immobilized on graphene showed a nearly 48-fold ECL amplification to detect chlorinated phenols (CPs) in water by using $\mathrm{K}_{2} \mathrm{~S}_{2} \mathrm{O}_{8}$ as a coreactant. ${ }^{179}$ The superior detection ability results from the high yield of the excited CQDS $\left(\mathrm{C}^{*^{+}}\right)$(Fig. 11e). ${ }^{180}$ The reaction mechanism diagram is shown in Fig. 11f and g. The ECL signal was decreased due to
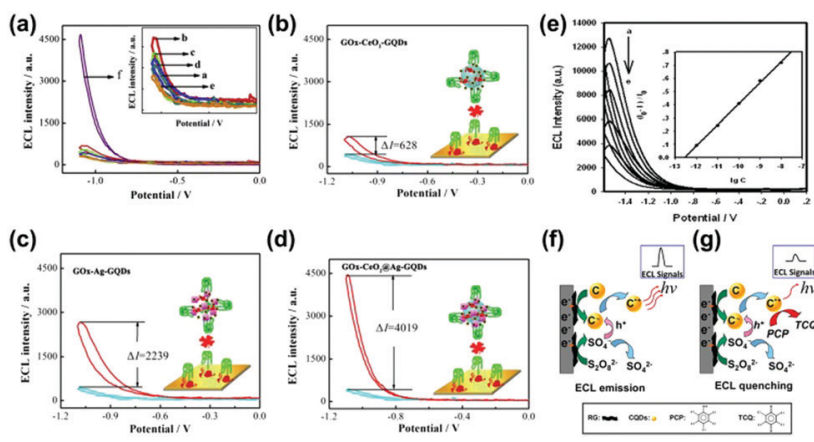

Fig. 11 (a) ECL response of different structures of biosensors; $a-d$ in the inset figure stand for bare glassy carbon electrode (GCE), electrodeposit of gold layers (Dp Au)/GCE, GOx/Dp Au/GCE, bovine serum albumin (BSA)/ GOx/Dp Au/GCE, Con A/BSA/GOx/Dp Au/GCE and (GOx-CeO $\mathrm{CeA}_{2} \mathrm{Ag}-$ GQDs/Con A/BSA/GOx/Dp Au/GCE). (b-d) The ECL responses of different biosensors' modified electrodes. ${ }^{178}$ (Reprinted with permission from Elsevier B.V., Copyright 2019.) (e) ECL intensity signal of CQDs/graphene in $\mathrm{S}_{2} \mathrm{O}_{8}{ }^{2-}$ solution $(\mathrm{pH}$ ) at gradient increasing concentrations of pentachlorophenol The mechanism diagram of (f) ECL emission and (g) ECL quenching. ${ }^{180}$ (Reprinted with permission from the American Chemical Society, Copyright 2013.) 
pentachlorophenol (PCP) on the CQDs surface oxidized by $\mathrm{C}^{*^{+}}$. Single-cell analysis is very important in bioscience and biomedical technology. To realize this objective, Yang and co-workers established an ECL analysis platform based on solid-state zinccoadsorbed CQDs hybrids for breast cancer cell detection. ${ }^{181}$ Through such a strategy, the determination of MDA-MB-231 and MCF-7 single cells with linear ranges of 1-18 and 1-12 cells has been realized, respectively.

\subsection{Chirality}

Chirality is defined as a property of a molecule by which it possesses a structure that is non-superimposable on its mirror image, which is one of the most pivotal factors to affect the optical properties of photosensitizer molecules. Chirality is ubiquitous in nature, not only in natural amino acids and DNA, but also in synthesized materials such as CDs.

Herranz, Martin and co-workers first reported the preparation of chiral GQDs from acidic exfoliation and oxidation of graphite in the presence of $(R)$ or $(S)$-2-phenyl-1-propanol. ${ }^{182}$ Pyrene was introduced to interact with GQDs. The GQDs-(S)/pyrene aggregates represented a positive cotton effect, while the GQDs$\mathrm{E}(R) /$ pyrene aggregates exhibited a negative cotton effect in the circular dichroism spectrum from 320 to $350 \mathrm{~nm}$. This result clearly revealed that the $(R)$ or $(S)$ configuration of 2-phenyl-1propanol has been introduced into the GQDs structure. Chiral CDs have also been synthesized by using D-/L-methionine, $\mathrm{D}-/ \mathrm{L}$-glucose, and $\mathrm{D}-/ \mathrm{L}$-glucosamine. ${ }^{183}$ The circular dichroism spectrum displayed a peak at around $210 \mathrm{~nm}$ in the positive direction for GQDs synthesized from D-methionine, while a different behavior was observed for GQDs from L-methionine. More interestingly, it was found that the chiral GQDs could affect the optical properties of azobenzene. Under UV light, azobenzene can react differently with D- and L-GQDs. Liu and co-workers synthesized chiral emitting CDs through a pyrolytic route using citric acid and $\mathrm{D}-\mathrm{L}-\mathrm{L} e n i c i l l a m i n e ~(\mathrm{D} / \mathrm{L}-\mathrm{Pen})$ (Fig. 12a). ${ }^{184}$ The chirality of Pen precursors was transferred to the CDs via an active surface passivation procedure (Fig. 12b). Fig. 12c shows anisotropic $\mathrm{D} / \mathrm{L}-\mathrm{Pen}$-CDs. Chiral CDs have been widely used for targeted therapy, modulating cellular functions, and photoluminescent enantioselective sensing. Similarly, chiral CDs have also been prepared by cyclic $\alpha$-amino acids ( $\mathrm{D}$ - or $\mathrm{L}$-enantiomer), and $\mathrm{D}$-/L-lysine as capping agents. ${ }^{185,186}$ Recently, the chirality mechanism of D-CQDs and L-CQDs has also been demonstrated (Fig. 12d). ${ }^{187}$ The spectra of $\mathrm{D} / \mathrm{L}$-CQDs and $\mathrm{D} / \mathrm{L}$-tryptophan $(\operatorname{Trp})$ were symmetrical in a certain range of wavelengths. Moreover, they were enantiomeric isomers (Fig. 12e and f). The chiral D-/L-CQDs were prepared using raw material $\mathrm{D}$-/L-tryptophan as both carbon and chiral sources. A symmetrical and opposite CDs spectrum has been observed for the $\mathrm{L}^{-}$and D-CQDs, showing three typical bands at 220,240 and $290 \mathrm{~nm}$. The former $220 \mathrm{~nm}$ signal is attributed to the inheritance of $\mathrm{L}-\mathrm{D} \mathrm{D}-\mathrm{Trp}$, and the latter two peaks are related to the inducement of the chiral environment. In detail, the $240 \mathrm{~nm}$ signal is ascribed to the $\pi-\pi^{*}$ conjugation of the $\mathrm{sp}^{2}$ carbon skeleton in $\mathrm{L}^{-}$and $\mathrm{D}$-CQDs, while the $290 \mathrm{~nm}$ signal is derived from the $\pi-\pi^{*}$ transition between the introduced

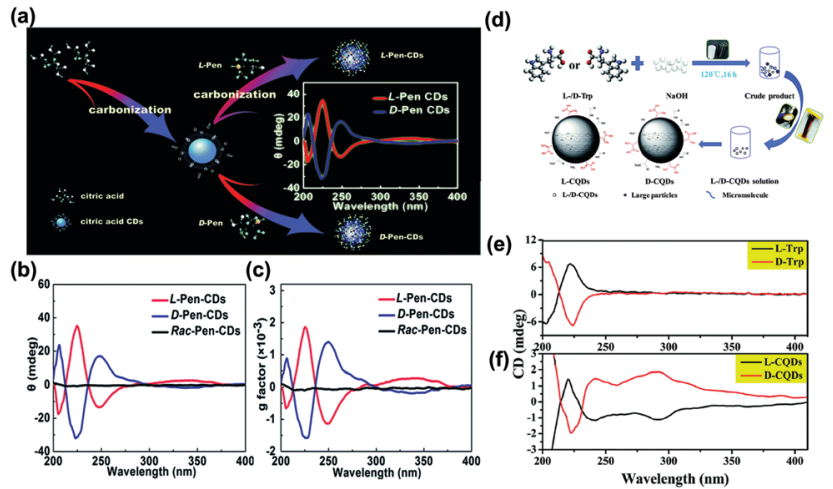

Fig. 12 (a) The schematic diagram of the formation of chiral light emitting CDs by citric acid and D/L-Pen as the carbon sources. (b) The circular dichroism spectra of the as-prepared CDs. (c) The corresponding anisotropy factors for all as-prepared CDs. ${ }^{184}$ (Reprinted with permission from the Royal Society of Chemistry, Copyright 2017.) (d) Schematic for the synthesis of L-/D-CQDs. (e) The circular dichroism spectra of L-/D-Trp. (f) The circular dichroism spectra of L-/D-CQDs. ${ }^{187}$ (Reprinted with permission from the Royal Society of Chemistry, Copyright 2017.).

indole. A clear understanding of the chiral mechanism provides great potential for their applications in chiral recognition and other fields.

\section{Summary and outlook}

This review provides an overview of the unique optical properties of CDs, including PL, NIR fluorescence, SERS, phosphorescence, CL, ECL, and chirality. As one of the emerging versatile materials, CDs with varied compositions and chemical structures provide tunable and unique optical properties. The distinguishable optical properties have been characterized by the corresponding spectroscopies. Despite realizing the outstanding optical properties of a large number of novel CDs, there are still some challenges to be addressed for guiding their further applications. In particular, more attention should be paid to improve the optical performance of the CDs.

Firstly, the synthesis of varied CDs with tunable compositions, sizes, shapes, crystallinity and electronic structures is essential to tailor their optical properties. Although many advances have been achieved to prepare multifunctional CDs, most reported strategies suffer from the shortcomings of time consuming, environmentally unfriendly and low quantity production. Therefore, it is urgent to develop an efficient, controllable and sustainable synthetic strategy to prepare CDs with high quality and a large volume.

Secondly, the PL properties especially with blue or green fluorescence have been broadly explored in most types of CDs. However, CDs with NIR fluorescence, phosphorescence, ECL, and SERS properties are rarely reported. CDs with these optical properties are more suitable for bio-imaging, light emitting and highly sensitive sensing applications. It is highly desirable to prepare unique CDs with such unique optical properties with a high efficiency for promising applications. 
Thirdly, the elucidation of the mechanism of the optical properties is still not clear. Up to now, much work has focused on the investigation of the PL mechanism of CDs, which has been attributed to the edge and surface states as well as the quantum confinement effect of CDs. However, there is still no strong evidence to propose a more universally acceptable PL mechanism of the CDs. Most importantly, the mechanisms of the other optical properties of CDs are much less investigated. It is necessary to conduct more theoretical and experimental research to better understand the mechanism of optical properties of CDs.

Finally, the hybridization of CDs with other functional materials is an efficient strategy to enhance their optical properties. In this regard, the modulation of the surface functionalization and interfacial effect of CDs-based hybrids is very important. In addition, much more attention including theoretical calculations and modern characterization techniques should be paid to the investigation of the correlation between the optical properties and their interfacial interactions.

We believe that the deep understanding of the optical properties of CDs will promote their promising applications in a large variety of fields. Furthermore, CDs are considered to be one of the most safe and eco-environmental materials. This makes CDs potential candidates for optical and photoelectronic applications.

\section{Conflicts of interest}

There are no conflicts to declare.

\section{Acknowledgements}

This work was financially supported by the National Natural Science Foundation of China $(21473068,21773080)$ and the Project of Jilin Province Science and Technology Development Plan Project (20180101295JC).

\section{Notes and references}

1 V. K. Voora and K. D. Jordan, Nano Lett., 2014, 14, 4602-4606.

2 G. Brinkmann, J. Goedgebeur and B. D. McKay, J. Chem. Inf. Model., 2012, 52, 2910-2918.

3 H. Li, Q. Zhou, F. Liu, W. Zhang, Z. Tan, H. Zhou, Z. Huang, S. Jiao and Y. Kuang, Appl. Catal., B, 2019, 255, 117755.

4 J. Lin, Y. Xu, J. Wang, B. Zhang, C. Wang, S. He and J. Wu, Chem. Eng. J., 2019, 373, 78-85.

5 Q. Liu, C. Zeng, Z. Xie, L. Ai, Y. Liu, Q. Zhou, J. Jiang, H. Sun and S. Wang, Appl. Catal., B, 2019, 254, 443-451.

6 F. Gorrini, R. Giri, C. E. Avalos, S. Tambalo, S. Mannucci, L. Basso, N. Bazzanella, C. Dorigoni, M. Cazzanelli, P. Marzola, A. Miotello and A. Bifone, ACS Appl. Mater. Interfaces, 2019, 11, 24412-24422.

7 O. Graydon, Nat. Photonics, 2019, 13, 438.
8 F. J. Hsieh, S. Sotoma, H. H. Lin, C. Y. Cheng, T. Y. Yu, C. L. Hsieh, C. H. Lin and H. C. Chang, ACS Appl. Mater. Interfaces, 2019, 11, 19774-19781.

9 M. Švec, P. Merino, Y. J. Dappe, C. González, E. Abad, P. Jelínek and J. A. Martín-Gago, Phys. Rev. B: Condens. Matter Mater. Phys., 2012, 86, 121407.

10 J. W. Feng, H. M. Ding and Y. Q. Ma, Carbon, 2015, 90, 34-43.

11 B. Kang, S. Wu, J. Ma, H. Ai and J. Y. Lee, Nanoscale, 2019, 11, 16599-16605.

12 F. Chang, L. Huang, C. Z. Guo, G. Xie, J. Li and Q. Diao, ACS Appl. Mater. Interfaces, 2019, 11, 35622-35629.

13 L. Bai, H. Yan, Y. Feng, W. Feng and L. Yuan, Chem. Eng. J., 2019, 373, 963-972.

14 M. Berenguel-Alonso, I. Ortiz-Gómez, B. Fernández, P. Couceiro, J. Alonso-Chamarro, L. F. Capitán-Vallvey, A. Salinas-Castillo and M. Puyol, Sens. Actuators, B, 2019, 296, 126613.

15 X. W. Hua, Y. W. Bao, J. Zeng and F. G. Wu, ACS Appl. Mater. Interfaces, 2019, 11, 32647-32658.

16 X. Y. Xu, R. Ray, Y. L. Gu, H. J. Ploehn, L. Gearheart, K. Raker and W. A. Scrivens, J. Am. Chem. Soc., 2004, 126, 12736-12737.

17 Y.-P. Sun, B. Zhou, Y. Lin, W. Wang, K. A. S. Fernando, P. Pathak, M. J. Meziani, B. A. Harruff, X. Wang, H. Wang, P. G. Luo, H. Yang, M. E. Kose, B. Chen, L. M. Veca and S.Y. Xie, J. Am. Chem. Soc., 2006, 128, 7756-7757.

18 B. B. Chen, M. L. Liu, C. M. Li and C. Z. Huang, Adv. Colloid Interface Sci., 2019, 270, 165-190.

19 M. L. Liu, B. B. Chen, C. M. Li and C. Z. Huang, Green Chem., 2019, 21, 449-471.

20 C. Xia, S. Zhu, T. Feng, M. Yang and B. Yang, Adv. Sci., 2019, 1901316.

21 J. Gao, M. Zhu, H. Huang, Y. Liu and Z. Kang, Inorg. Chem. Front., 2019, 4, 1963-1986.

22 C. Hu, M. Li, J. Qiu and Y. Sun, Chem. Soc. Rev., 2019, 48, 2315-2337.

23 J. B. Essner and G. A. Baker, Environ. Sci.: Nano, 2017, 4, 1216-1263.

24 B. B. Chen, Z. X. Liu, W. C. Deng, L. Zhan, M. L. Liu and C. Z. Huang, Green Chem., 2016, 18, 5127-5132.

25 M. L. Liu, L. Yang, R. S. Li, B. B. Chen, H. Liu and C. Z. Huang, Green Chem., 2017, 19, 3611-3617.

26 H. Li, Z. Kang, Y. Liu and S.-T. Lee, J. Mater. Chem., 2012, 22, 24230.

27 X. Li, H. Wang, Y. Shimizu, A. Pyatenko, K. Kawaguchi and N. Koshizaki, Chem. Commun., 2011, 47, 932-934.

28 S. L. Hu, K. Y. Niu, J. Sun, J. Yang, N. Q. Zhao and X. W. Du, J. Mater. Chem., 2009, 19, 484-488.

29 Q. L. Zhao, Z. L. Zhang, B. H. Huang, J. Peng, M. Zhang and D. W. Pang, Chem. Commun., 2008, 5116-5118.

30 L. Lin and S. Zhang, Chem. Commun., 2012, 48, 10177-10179.

31 W. Wang, C. Damm, J. Walter, T. J. Nacken and W. Peukert, Phys. Chem. Chem. Phys., 2016, 18, 466-475.

32 A. Sciortino, A. Cayuela, M. L. Soriano, F. M. Gelardi, M. Cannas, M. Valcárcel and F. Messina, Phys. Chem. Chem. Phys., 2016, 18, 466-475. 
33 S. Tao, T. Feng, C. Zheng, S. Zhu and B. Yang, J. Phys. Chem. Lett., 2019, 10, 5182-5188.

34 F. Wang, S. Pang, L. Wang, Q. Li, M. Kreiter and C. Y. Liu, Chem. Mater., 2010, 22, 4528-4530.

35 J. Zong, Y. Zhu, X. Yang, J. Shen and C. Li, Chem. Commun., 2011, 47, 764-766.

36 D. Sun, R. Ban, P. H. Zhang, G. H. Wu, J. R. Zhang and J. J. Zhu, Carbon, 2013, 64, 424-434.

37 W. Guan, W. Gu, L. Ye, C. Guo, S. Su, P. Xu and M. Xue, Int. J. Nanomed., 2014, 9, 5071-5078.

38 A. B. Bourlinos, R. Zbořil, J. Petr, A. Bakandritsos, M. Krysmann and E. P. Giannelis, Chem. Mater., 2011, 24, 6-8.

39 L. Cao, X. Wang, M. J. Meziani, F. Lu, H. Wang, P. G. Luo, Y. Lin, B. A. Harruff, L. M. Veca, D. Murray, S. Y. Xie and Y. P. Sun, J. Am. Chem. Soc., 2007, 129, 11318-11319.

40 K. Chen, W. Qing, W. Hu, M. Lu, Y. Wang and X. Liu, Spectrochim. Acta, Part A, 2019, 213, 228-234.

41 K. Lu, C. Li, H. Z. Wang, Y. L. Li, Y. Zhu and Y. Ouyang, J. Photochem. Photobiol., B, 2019, 197, 111504.

42 B. Das, A. Girigoswami, P. Pal and S. Dhara, Mater. Sci. Eng., C, 2019, 102, 427-436.

43 Z. Zhang, X. Wang, X. Zhang, P. Yu, L. Geng and S. Shi, Mater. Res. Bull., 2018, 99, 225-231.

44 M. Hashemi, J. Mohammadi, M. Omidi, H. D. C. Smyth, B. Muralidharan, T. E. Milner, A. Yadegari, D. Ahmadvand, M. Shalbaf and L. Tayebi, Mater. Sci. Eng., C, 2019, 103, 109860.

45 Y. Dong, G. Li, N. Zhou, R. Wang, Y. Chi and G. Chen, Anal. Chem., 2012, 84, 8378-8382.

46 Y. Dong, H. Pang, H. B. Yang, C. Guo, J. Shao, Y. Chi, C. M. Li and T. Yu, Angew. Chem., Int. Ed., 2013, 52, 7800-7804.

47 R. Liu, D. Wu, X. Feng and K. Müllen, J. Am. Chem. Soc., 2011, 133, 15221-15223.

48 V. Tucureanu, A. Matei and A. M. Avram, Crit. Rev. Anal. Chem., 2016, 46, 502-520.

49 T. Susi, T. Pichler and P. Ayala, Beilstein J. Nanotechnol., 2015, 6, 177-192.

50 A. Philippidis, A. Spyros, D. Anglos, A. B. Bourlinos, R. Zbořil and E. P. Giannelis, J. Nanopart. Res., 2013, 15, 1777.

51 H. Ming, Z. Ma, Y. Liu, K. Pan, H. Yu, F. Wang and Z. Kang, Dalton Trans., 2012, 41, 9526-9531.

52 J. Peng, W. Gao, B. K. Gupta, Z. Liu, R. Romero-Aburto, L. Ge, L. Song, L. B. Alemany, X. Zhan, G. Gao, S. A. Vithayathil, B. A. Kaipparettu, A. A. Marti, T. Hayashi, J. J. Zhu and P. M. Ajayan, Nano Lett., 2012, 12, 844-849.

53 C. Lin, Y. Zhuang, W. Li, T. L. Zhou and R. J. Xie, Nanoscale, 2019, 11, 6584-6590.

54 X. Jia, J. Li and E. Wang, Nanoscale, 2012, 4, 5572-5575.

55 L. Tang, R. Ji, X. Cao, J. Lin, H. Jiang, X. Li, K. S. Teng, C. M. Luk, S. Zeng, J. Hao and S. P. Lau, ACS Nano, 2012, 6, 5102-5110.

56 C. Zhu, S. Yang, J. Sun, P. He, N. Yuan, J. Ding, R. Mo, G. Wang, G. Ding and X. Xie, Synth. Met., 2015, 209, 468-472.
57 S. He, M. J. Turnbull, Y. Nie, X. Sun and Z. Ding, Surf. Sci., 2018, 676, 51-55.

58 M. P. More, P. H. Lohar, A. G. Patil, P. O. Patil and P. K. Deshmukh, Mater. Chem. Phys., 2018, 220, 11-22.

59 J. Ren, F. Weber, F. Weigert, Y. Wang, S. Choudhury, J. Xiao, I. Lauermann, U. Resch-Genger, A. Bande and T. Petit, Nanoscale, 2019, 11, 2056-2064.

60 V. Kumar, V. Singh, S. Umrao, V. Parashar, S. Abraham, A. K. Singh, G. Nath, P. S. Saxena and A. Srivastava, RSC Adv., 2014, 4, 21101.

61 L. Zhang, L. Li, C. Ma, S. Ge, M. Yan and C. Bian, Sens. Actuators, B, 2015, 221, 799-806.

62 J. P. Guin, S. K. Guin, T. Debnath and H. N. Ghosh, Carbon, 2016, 109, 517-528.

63 H. Xu, S. Zhou, L. Xiao, S. Li, T. Song, Y. Wang and Q. Yuan, Carbon, 2015, 87, 215-225.

64 J. Cheng, C.-F. Wang, Y. Zhang, S. Yang and S. Chen, RSC Adv., 2016, 6, 37189-37194.

65 Y. Zhang, J. Zhang, J. Zhang, S. Lin, Y. Huang, R. Yuan, X. Liang and W. Xiang, Dyes Pigm., 2017, 140, 122-130.

66 A. Bhati, S. R. Anand, D. Saini, P. Khare, P. Dubey and S. K. Sonkar, New J. Chem., 2018, 42, 19548-19556.

67 F. Huo, W. Liang, Y. Tang, W. Zhang, X. Liu, D. Pei, H. Wang, W. Jia, P. Jia and F. Yang, J. Mater. Sci., 2019, 54, 6815-6825.

68 J. Zhao, F. Li, S. Zhang, Y. An and S. Sun, New J. Chem., 2019, 43, 6332-6342.

69 P. Dong, B. P. Jiang, W. Q. Liang, Y. Huang, Z. Shi and X. C. Shen, Inorg. Chem. Front., 2017, 4, 712-718.

70 N. Far'ain Md Noor, M. A. Saiful Badri, M. M. Salleh and A. A. Umar, Opt. Mater., 2018, 83, 306-314.

71 K. Zhao, X. Zheng, H. Zhang, M. Xu, S. Wang, Q. Yang and C. Xiong, J. Alloys Compd., 2019, 793, 613-619.

72 S. Zhu, Y. Song, J. Wang, H. Wan, Y. Zhang, Y. Ning and B. Yang, Nano Today, 2017, 13, 10-14.

73 F. Yan, Z. Sun, H. Zhang, X. Sun, Y. Jiang and Z. Bai, Microchim. Acta, 2019, 186, 583.

74 L. Ge, N. Pan, J. Jin, P. Wang, G. E. LeCroy, W. Liang, L. Yang, L. R. Teisl, Y. Tang and Y. P. Sun, J. Phys. Chem. C, 2018, 122, 21667-21676.

75 M. Yang, H. Mei, Y. Shen, K. Wu, D. Pan, S. Liu, T. Zhang and Y. Zhang, Chem. - Eur. J., 2019, 25, 1-10.

76 H. Li, X. He, Z. Kang, H. Huang, Y. Liu, J. Liu, S. Lian, C. H. Tsang, X. Yang and S. T. Lee, Angew. Chem., Int. Ed., 2010, 49, 4430-4434.

77 R. Q. Zhang, E. Bertran and S. T. Lee, Diam. Relat. Mater., 1998, 7, 1663-1668.

78 Z. X. Liu, H. Y. Zou, N. Wang, T. Yang, Z. L. Peng, J. Wang, N. Li and C. Z. Huang, Sci. China: Chem., 2018, 61, 490-496.

79 M. F. Budyka, Spectrochim. Acta, Part A, 2019, 207, 1-5.

80 T. F. Yeh, W. L. Huang, C. J. Chung, I. T. Chiang, L. C. Chen, H. Y. Chang, W. C. Su, C. Cheng, S. J. Chen and H. Teng, J. Phys. Chem. Lett., 2016, 7, 2087-2092.

81 G. Rajender and P. K. Giri, J. Mater. Chem. C, 2016, 4, 10852-10865.

82 G. Rajender, U. Goswami and P. K. Giri, J. Colloid Interface Sci., 2019, 541, 387-398. 
83 J. Yu, C. Liu, K. Yuan, Z. Lu, Y. Cheng, L. Li, X. Zhang, P. Jin, F. Meng and H. Liu, Nanomaterials, 2018, 8, 233.

84 Q. Xu, Q. Zhou, Z. Hua, Q. Xue, C. Zhang, X. Wang, D. Pan and M. Xiao, ACS Nano, 2013, 7, 10654-10661.

85 Q. Fang, Y. Dong, Y. Chen, C.-H. Lu, Y. Chi, H.-H. Yang and T. Yu, Carbon, 2017, 118, 319-326.

86 H. Ding, S. B. Yu, J. S. Wei and H. M. Xiong, ACS Nano, 2016, 10, 484-491.

87 D. Bera, L. Qian, T.-K. Tseng and P. H. Holloway, Materials, 2010, 3, 2260-2345.

88 K. K. Chan, C. Yang, Y.-H. Chien, N. Panwar and K.-T. Yong, New J. Chem., 2019, 43, 4734-4744.

89 H. Wang, C. Sun, X. Chen, Y. Zhang, V. L. Colvin, Q. Rice, J. Seo, S. Feng, S. Wang and W. W. Yu, Nanoscale, 2017, 9, 1909-1915.

90 M. A. Sk, A. Ananthanarayanan, L. Huang, K. H. Lim and P. Chen, J. Mater. Chem. C, 2014, 2, 6954-6960.

91 H. Kalita, J. Mohapatra, L. Pradhan, A. Mitra, D. Bahadur and M. Aslam, RSC Adv., 2016, 6, 23518-23524.

92 L. Zhou, J. Geng and B. Liu, Part. Part. Syst. Charact., 2013, 30, 1086-1092.

93 Y. H. Yuan, Z. X. Liu, R. S. Li, H. Y. Zou, M. Lin, H. Liu and C. Z. Huang, Nanoscale, 2016, 8, 6770-6776.

94 M. Madhu, T. H. Chen and W. L. Tseng, J. Colloid Interface Sci., 2019, 556, 120-127.

95 S. Yang, J. Sun, X. Li, W. Zhou, Z. Wang, P. He, G. Ding, X. Xie, Z. Kang and M. Jiang, J. Mater. Chem. A, 2014, 2, 8660 .

96 X. Sun, H. Liu, L. Yang, X. Wang, W. Yang, M. Wei, X. Liu, J. Cao, J. Yang and S. G. Xing, Nanomaterials, 2018, 8, 635.

97 H. Tetsuka, A. Nagoya, T. Fukusumi and T. Matsui, Adv. Mater., 2016, 28, 4632-4638.

98 S. Zhu, Q. Meng, L. Wang, J. Zhang, Y. Song, H. Jin, K. Zhang, H. Sun, H. Wang and B. Yang, Angew. Chem., Int. Ed., 2013, 52, 3953-3957.

99 Y. Dong, H. Pang, H. B. Yang, C. Guo, J. Shao, Y. Chi, C. M. Li and T. Yu, Angew. Chem., Int. Ed., 2013, 52, 7800-7804.

100 J. Zong, X. Yang, A. Trinchi, S. Hardin, I. Cole, Y. Zhu, C. Li, T. Muster and G. Wei, Nanoscale, 2013, 5, 11200-11206.

101 C. Liu, L. Bao, B. Tang, J. Y. Zhao, Z. L. Zhang, L. H. Xiong, J. Hu, L. L. Wu and D. W. Pang, Small, 2016, 12, 4702-4706.

102 Q. Xu, R. Su, Y. Chen, S. T. Sreenivasan, N. Li, X. Zheng, J. Zhu, H. Pan, W. Li, C. Xu, Z. Xia and L. Dai, ACS Appl. Nano. Mater., 2018, 1, 1886-1893.

103 Y. Xie, J. Zheng, Y. Wang, J. Wang, Y. Yang, X. Liu and Y. Chen, Nanotechnology, 2019, 30, 085406.

104 S. Li, Y. Li, J. Cao, J. Zhu, L. Fan and X. Li, Anal. Chem., 2014, 86, 10201-10207.

105 S. Kalytchuk, K. Poláková, Y. Wang, J. P. Froning, K. Cepe, A. L. Rogach and R. Zbořil, ACS Nano, 2017, 11, 1432-1442.

106 Y. Li, G. Bai, S. Zeng and J. Hao, ACS Appl. Mater. Interfaces, 2019, 11, 4737-4744.
107 D. Li, P. Jing, L. Sun, Y. An, X. Shan, X. Lu, D. Zhou, D. Han, D. Shen, Y. Zhai, S. Qu, R. Zboril and A. L. Rogach, Adv. Mater., 2018, 30, e1705913.

108 Y. Zhan, T. Geng, Y. Liu, C. Hu, X. Zhang, B. Lei, J. Zhuang, X. Wu, D. Huang, G. Xiao and B. Zou, ACS Appl. Mater. Interfaces, 2018, 10, 27920-27927.

109 L. Cao, X. Wang, M. J. Meziani, F. Lu, H. Wang, P. G. Luo, Y. Lin, B. A. Harruff, L. M. Veca, D. Murray, S. Y. Xie and Y. P. Sun, J. Am. Chem. Soc., 2007, 129, 11318-11319.

110 Q. Liu, B. Guo, Z. Rao, B. Zhang and J. R. Gong, Nano Lett., 2013, 13, 2436-2441.

111 Y. Cui, Z. Hu, C. Zhang and X. Liu, J. Mater. Chem. B, 2014, 2, 6947-6953.

112 D. Lu, Y. Tang, J. Gao, Y. Chen and Q. Wang, Dyes Pigm., 2019, 165, 287-293.

113 H. D. Ha, M.-H. Jang, F. Liu, Y.-H. Cho and T. S. Seo, Carbon, 2015, 81, 367-375.

114 S. Zhuo, M. Shao and S. T. Lee, ACS Nano, 2012, 6, 1059-1064.

115 R. Xie, L. Zhang, H. Xu, Y. Zhong, X. Sui and Z. Mao, Chem. Eng. J., 2017, 310, 79-90.

116 B. Kong, A. Zhu, C. Ding, X. Zhao, B. Li and Y. Tian, Adv. Mater., 2012, 24, 5844-5848.

117 J. Shen, Y. Zhu, C. Chen, X. Yang and C. Li, Chem. Commun., 2011, 47, 2580-2582.

118 S. Y. Ding, E. M. You, Z. Q. Tian and M. Moskovits, Chem. Soc. Rev., 2017, 46, 4042-4076.

119 M. F. Cardinal, E. Vander Ende, R. A. Hackler, M. O. McAnally, P. C. Stair, G. C. Schatz and R. P. Van Duyne, Chem. Soc. Rev., 2017, 46, 3886-3903.

120 Z. Jie, Y. Zenghe, Z. Xiaolei and Z. Yong, Opt. Express, 2018, 26, 23534-23539.

121 X. Jiang, D. Yin, M. Yang, J. Du, W. Wang, L. Zhang, L. Yang, X. Han and B. Zhao, Appl. Surf. Sci., 2019, 487, 938-944.

122 R. Asapu, R. G. Ciocarlan, N. Claes, N. Blommaerts, M. Minjauw, T. Ahmad, J. Dendooven, P. Cool, S. Bals, S. Denys, C. Detavernier, S. Lenaerts and S. W. Verbruggen, ACS Appl. Mater. Interfaces, 2017, 9, 41577-41585.

123 H. Cheng, Y. Zhao, Y. Fan, X. Xie, L. Qu and G. Shi, ACS Nano, 2012, 6, 2237-2244.

124 C. Li, H. Wang, Y. Luo, G. Wen and Z. Jiang, Food Chem., 2019, 289, 531-536.

125 C. Li, P. Fan, A. Liang and Z. Jiang, Mater. Sci. Eng., C, 2019, 99, 1399-1406.

126 X. Feng, C. Li, A. Liang, Y. Luo and Z. Jiang, Nanomaterials, 2019, 9, 480.

127 Y. Fan, H. Cheng, C. Zhou, X. Xie, Y. Liu, L. Dai, J. Zhang and L. Qu, Nanoscale, 2012, 4, 1776-1781.

128 J. Jin, S. Zhu, Y. Song, H. Zhao, Z. Zhang, Y. Guo, J. Li, W. Song, B. Yang and B. Zhao, ACS Appl. Mater. Interfaces, 2016, 8, 27956-27965.

129 H. Zhao, Y. Guo, S. Zhu, Y. Song, J. Jin, W. Ji, W. Song, B. Zhao, B. Yang and Y. Ozaki, Appl. Surf. Sci., 2017, 410, 42-50. 
130 Z. Mao, W. Song, X. Xue, W. Ji, Z. Li, L. Chen, H. Mao, H. Lv, X. Wang, J. R. Lombardi and B. Zhao, J. Phys. Chem. C, 2012, 116, 14701-14710.

131 G. Qi, Y. Zhang, S. Xu, C. Li, D. Wang, H. Li and Y. Jin, Anal. Chem., 2018, 90, 13356-13364.

132 X. Zhang and X. Du, ACS Appl. Mater. Interfaces, 2016, 8, 1033-1040.

133 P. Y. Cao, Y. L. Liu, Z. T. Lin, J. F. Huang, W. Z. Chen, J. H. Liang, W. Zhou and G. B. Jiang, Carbon, 2017, 118, 625-633.

134 D. Liu, X. Chen, Y. Hu, T. Sun, Z. Song, Y. Zheng, Y. Cao, Z. Cai, M. Cao, L. Peng, Y. Huang, L. Du, W. Yang, G. Chen, D. Wei, A. T. S. Wee and D. Wei, Nat. Commun., 2018, 9, 193.

135 G. Baryshnikov, B. Minaev and H. Ågren, Chem. Rev., 2017, 117, 6500-6537.

136 Y. Deng, D. Zhao, X. Chen, F. Wang, H. Song and D. Shen, Chem. Commun., 2013, 49, 5751-5753.

137 Q. Li, M. Zhou, Q. Yang, Q. Wu, J. Shi, A. Gong and M. Yang, Chem. Mater., 2016, 28, 8221-8227.

138 Q. Li, M. Zhou, M. Yang, Q. Yang, Z. Zhang and J. Shi, Nat. Commun., 2018, 9, 734.

139 J. Tan, Y. Ye, X. Ren, W. Zhao and D. Yue, J. Mater. Chem. C, 2018, 6, 7890-7895.

140 Q. Li, M. Zhou, M. Yang, Q. Yang, Z. Zhang and J. Shi, Nat. Commun., 2018, 9, 734.

141 L. Zhang, R. Zhang, P. Cui, W. Cao and F. Gao, Chem. Commun., 2013, 49, 8102-8104.

142 L. Q. Bai, N. Xue, X. R. Wang, W. Y. Shi and C. Lu, Nanoscale, 2017, 9, 6658-6664.

143 C. Wang, Y. Chen, T. Hu, Y. Chang, G. Ran, M. Wang and Q. Song, Nanoscale, 2019, 11, 11967-11974.

144 W. Li, W. Zhou, Z. Zhou, H. Zhang, X. Zhang, J. Zhuang, Y. Liu, B. Lei and C. Hu, Angew. Chem., Int. Ed., 2019, 58, $7278-7283$

145 K. Jiang, Y. Wang, C. Cai and H. Lin, Adv. Mater., 2018, 30, e1800783.

146 K. Jiang, Y. Wang, X. Gao, C. Cai and H. Lin, Angew. Chem., Int. Ed., 2018, 57, 6216-6220.

147 P. Long, Y. Feng, C. Cao, Y. Li, J. Han, S. Li, C. Peng, Z. Li and W. Feng, Adv. Funct. Mater., 2018, 28, 1800791.

148 S. Tao, S. Lu, Y. Geng, S. Zhu, S. A. T. Redfern, Y. Song, T. Feng, W. Xu and B. Yang, Angew. Chem., Int. Ed., 2018, 57, 2393-2398.

149 L. Li, Y. Chen and J. Zhu, Anal. Chem., 2017, 89, 358-371.

150 D. M. Wang, K. L. Lin and C. Huang, Luminescence, 2019, 34, 4-22.

151 J. Zhang, X. Lu, D. Tang, S. Wu, X. Hou, J. Liu and P. Wu, ACS Appl. Mater. Interfaces, 2018, 10, 40808-40814.

152 Z. Lin, W. Xue, H. Chen and J. M. Lin, Anal. Chem., 2011, 83, 8245-8251.

153 R. Sha, A. Gopalakrishnan, K. V. Sreenivasulu, V. V. S. S. Srikanth and S. Badhulika, J. Alloys Compd., 2019, 794, 26-34.

154 J. T. Cao, W. S. Zhang, H. Wang, S. H. Ma and Y. M. Liu, Spectrochim. Acta, Part A, 2019, 219, 281-287.
155 J. Wang, M. M. Hassan, W. Ahmad, T. Jiao, Y. Xu, H. Li, Q. Ouyang, Z. Guo and Q. Chen, Sens. Actuators, B, 2019, 285, 302-309.

156 S. Rafiei, M. Dadmehr, M. Hosseini, H. A. Kermani and M. R. Ganjali, Methods Appl. Fluoresc., 2019, 7, 025001.

157 S. N. Shah, H. Li and J. M. Lin, Talanta, 2016, 153, 23-30.

158 H. Zhang, X. Zhang and S. Dong, Anal. Chem., 2015, 87, 11167-11170.

159 H. Abdolmohammad-Zadeh and E. Rahimpour, Sens. Actuators, B, 2016, 225, 258-266.

160 M. Amjadi, J. L. Manzoori, T. Hallaj and T. Shahbazsaghir, Microchim. Acta, 2017, 184, 1587-1593.

161 T. Hallaj, M. Amjadi, Z. Song and R. Bagheri, Mikrochim. Acta, 2017, 185, 67.

162 L. Gao, L. Ju and H. Cui, J. Mater. Chem. C, 2017, 5, 7753-7758.

163 M. Vazquez-Gonzalez, W. C. Liao, R. Cazelles, S. Wang, X. Yu, V. Gutkin and I. Willner, ACS Nano, 2017, 11, 3247-3253.

164 B. Shi, Y. Su, Y. Duan, S. Chen and W. Zuo, Microchim. Acta, 2019, 186, 397.

165 S. Dong, Z. Yuan, L. Zhang, Y. Lin and C. Lu, Anal. Chem., 2017, 89, 12520-12526.

166 D. Li, F. Nie, T. Tang and K. Tian, Microchim. Acta, 2018, 185, 431.

167 M. M. Richter, Chem. Rev., 2004, 104, 3003-3036.

168 W. Miao, Chem. Rev., 2008, 108, 2506-2553.

169 H. Zhu, X. Wang, Y. Li, Z. Wang, F. Yang and X. Yang, Chem. Commun., 2009, 5118-5120.

170 L.-L. Li, J. Ji, R. Fei, C.-Z. Wang, Q. Lu, J.-R. Zhang, L.-P. Jiang and J.-J. Zhu, Adv. Funct. Mater., 2012, 22, 2971-2979.

171 H. Xie, F. Bai, Y. Fu, H. Zhu, K. Yan, K. Mao, P. K. Chu, L. Liu and X. Wu, Carbon, 2019, 147, 532-539.

172 Z. Guo, T. Hao, S. Du, B. Chen, Z. Wang, X. Li and S. Wang, Biosens. Bioelectron., 2013, 44, 101-107.

173 H. F. Zhao, R. P. Liang, J. W. Wang and J. D. Qiu, Chem. Commun., 2015, 51, 12669-12672.

174 P. Zhang, Z. Xue, D. Luo, W. Yu, Z. Guo and T. Wang, Anal. Chem., 2014, 86, 5620-5623.

175 C. Xiong, W. Liang, H. Wang, Y. Zheng, Y. Zhuo, Y. Chai and R. Yuan, Chem. Commun., 2016, 52, 5589-5592.

176 W. J. Niu, R. H. Zhu, S. Cosnier, X. J. Zhang and D. Shan, Anal. Chem., 2015, 87, 11150-11156.

177 C. Wang, J. Qian, K. Wang, M. Hua, Q. Liu, N. Hao, T. You and X. Huang, ACS Appl. Mater. Interfaces, 2015, 7, 26865-26873.

178 F. Zuo, C. Zhang, H. Zhang, X. Tan, S. Chen and R. Yuan, Electrochim. Acta, 2019, 294, 76-83.

179 X. L. Cai, B. Zheng, Y. Zhou, M. R. Younis, F. B. Wang, W. M. Zhang, Y. G. Zhou and X. H. Xia, Chem. Sci., 2018, 9, 6080-6084.

180 S. Yang, J. Liang, S. Luo, C. Liu and Y. Tang, Anal. Chem., 2013, 85, 7720-7725.

181 Y. Qiu, B. Zhou, X. Yang, D. Long, Y. Hao and P. Yang, ACS Appl. Mater. Interfaces, 2017, 9, 16848-16856. 
182 M. Vazquez-Nakagawa, L. Rodriguez-Perez, M. A. Herranz and N. Martin, Chem. Commun., 2016, 52, 665-668.

183 M. J. Deka and D. Chowdhury, RSC Adv., 2017, 7, 53057-53063.

184 M. Yuan, Y. Guo, J. Wei, J. Li, T. Long and Z. Liu, RSC Adv., 2017, 7, 49931-49936.
185 F. Ostadhossein, G. Vulugundam, S. K. Misra, I. Srivastava and D. Pan, Bioconjugate Chem., 2018, 29, 3913-3922.

86 F. Copur, N. Bekar, E. Zor, S. Alpaydin and H. Bingol, Sens. Actuators, B, 2019, 279, 305-312.

187 Y. Wei, L. Chen, J. Wang, X. Liu, Y. Yang and S. Yu, RSC Adv., 2019, 9, 3208-3214. 